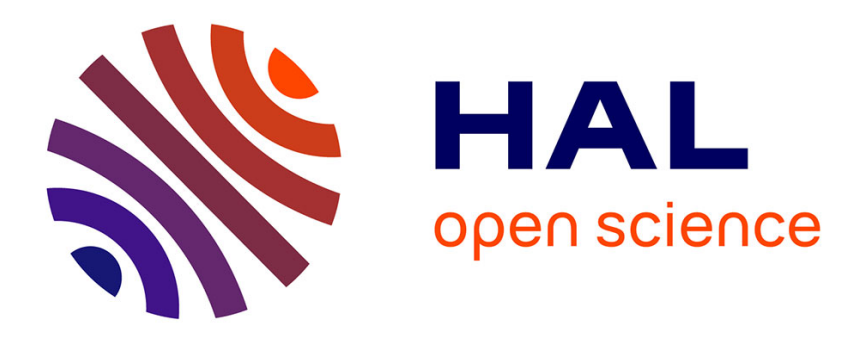

\title{
Superconducting properties of vacuum in strong magnetic field
}

\author{
Maxim N. Chernodub
}

\section{To cite this version:}

Maxim N. Chernodub. Superconducting properties of vacuum in strong magnetic field. International Journal of Modern Physics D, 2014, 23, pp.1430009. 10.1142/S0218271814300092 . hal-01058420

\section{HAL Id: hal-01058420 \\ https://hal.science/hal-01058420}

Submitted on 9 Sep 2014

HAL is a multi-disciplinary open access archive for the deposit and dissemination of scientific research documents, whether they are published or not. The documents may come from teaching and research institutions in France or abroad, or from public or private research centers.
L'archive ouverte pluridisciplinaire HAL, est destinée au dépôt et à la diffusion de documents scientifiques de niveau recherche, publiés ou non, émanant des établissements d'enseignement et de recherche français ou étrangers, des laboratoires publics ou privés. 


\title{
Superconducting properties of vacuum in strong magnetic field
}

\author{
M. N. CHERNODUB* \\ CNRS, Laboratoire de Mathématiques et Physique Théorique, Université François-Rabelais, \\ Fédération Denis Poisson - CNRS, Parc de Grandmont, Université de Tours, 37200 France \\ Department of Physics and Astronomy, University of Gent, Krijgslaan 281, S9, B-9000 Gent, \\ Belgium \\ maxim.chernodub@lmpt.univ-tours.fr
}

Received: 13 March 2014

Accepted: 24 March 2014

Published: 23 April 2014

\begin{abstract}
We discuss superconducting phases of vacuum induced by strong magnetic field in the Electroweak model and in Quantum Chromodynamics at zero temperature. In these phases the vacuum behaves as an anisotropic inhomogeneous superconductor which supports superconductivity along the axis of the magnetic field while in the transversal directions the superconductivity does not exist. The magnetic-field-induced anisotropic superconductivity appears as a result of condensation of electrically charged spin-one particles, which are elementary $W$ bosons in the case of the Electroweak model and composite quark-antiquark pairs with quantum numbers of $\rho$ mesons in the case of QCD. Due to the anisotropic nature of superconductivity the Meissner effect is absent. Intrinsic inhomogeneities of the superconducting ground state are characterized by ensembles of certain topological vortices in an analogy with a mixed Abrikosov state of a type-II superconductivity.
\end{abstract}

Keywords: Quantum Chromodynamics, Electroweak Model, Strong Magnetic Field, Superconductivity

PACS numbers: 11.15.Kc, 12.15.y, 12.38.-t, 13.40.-f, 25.75.-q, 74.90.+n

\section{Published in (link to the publisher's page): \\ International Journal of Modern Physics D 23, 1430009 (2014) DOI: $10.1142 / S 0218271814300092$}

\footnotetext{
*On leave from ITEP, Moscow, Russia.
} 


\section{Contents}

1. Vortex ground state in the Ginzburg-Landau model . . . . . . . . . . 5

1.1. Lagrangian and equations of motion . . . . . . . . . . . 5

1.2. Basic properties of an ordinary superconductor . . . . . . . . . 6

1.2.1. First London equation: isotropic superconductivity . . . . . . 6

1.2.2. Second London Equation: the Meissner effect . . . . . . . . 6

1.2.3. Destructive role of magnetic field . . . . . . . . . . . . 7

1.2.4. Abrikosov lattice of vortices in the mixed state . . . . . . 7

1.3. Vortex lattices . . . . . . . . . . . . . . . . 8

1.4. Reentrant superconductivity in strong magnetic field . . . . . . . . . 12

2. Electroweak model in strong magnetic field . . . . . . . . . . . . . . . . . 13

2.1. Structure of the ground state . . . . . . . . . . . . . . . 13

2.1.1. Lagrangian . . . . . . . . . . . . . . . . . 13

2.1.2. Instability of the ground state in background magnetic field . 14

2.1.3. Equations of motion and energy density . . . . . . . . . . 15

2.1.4. Phase structure in strong magnetic field: a brief overview . . 16

2.2. Superconducting vacuum near the first critical magnetic field . . . . 17

2.2.1. $W$ condensate and energy density . . . . . . . . . . . 17

2.2.2. Neutral and Higgs condensates . . . . . . . . . . . . . 20

2.2.3. Kaleidoscopic vortex structure of the $W$ and $Z$ condensates . 21

2.2.4. Nondissipative transport in the ground state . . . . . . . . 23

2.3. Symmetry restoration at the second critical magnetic field . . . . . 25

2.3.1. Structure of the symmetry restored phase . . . . . . . . 25

2.3.2. Vortex solutions . . . . . . . . . . . . . 27

3. Superconducting phase in QCD . . . . . . . . . . . . . . 29

3.1. Effective mean-field approach to QCD in strong magnetic field . . . 30

3.1.1. Electrodynamics of $\rho$ mesons . . . . . . . . . . . 30

3.1.2. The $\rho$-meson condensation due to strong magnetic field . . . 31

3.1.3. Equations of motion of the effective model . . . . . . . . . . . 32

3.1.4. Induced condensates and energy density of the ground state . 33

3.1.5. Superconductor vortices: the lattice structure . . . . . . . . . 35

3.1.6. Charged vector condensate and energy density . . . . . . . 36

3.1.7. Electric currents and superconductivity . . . . . . . . . . 38

3.1.8. Anisotropic superconductivity of the ground state . . . . . . 40

3.1.9. Symmetries of QCD ground state in the superconducting phase 42

3.2. Beyond the mean field . . . . . . . . . . . . . . . . . . . 44

3.2.1. Acoustic vibrations of the vortex lattice . . . . . . . . . . 44

3.2.2. Melting of the vortex lattice: results of numerical simulations 45

3.2.3. Nature of the transition to the superconducting ground state 47

4. Conclusions . . . . . . . . . . . . . . . . . . . . . . 49 
Strong magnetic fields may lead to many unusual phenomena both in dense matter and in the quantum vacuum. There are various QED effects associated with a critical magnetic field $B^{\mathrm{QED}}=m_{e}^{2} c^{3} / \hbar e \approx 4.4 \times 10^{9} \mathrm{~T}$ at which the splitting between zeroth and first Landau electron's levels exceeds the rest energy of an electron, $m_{e} c^{2}$. Such strong fields make the QED vacuum optically birefringent, ${ }^{1}$ leading to distortion and magnification of images (a magnetic lens effect), splitting and merging of photons and affecting strongly atomic spectra. ${ }^{2}$

At much stronger magnetic fields of the order of the QCD scale, $B \sim 10^{16} \mathrm{~T}$ various other interesting effects may happen. The magnetic fields of this strength may be created on Earth in heavy-ion collisions at the Large Hadron Collider at CERN in Europe and at Relativistic Heavy-Ion Collider at Brookhaven National Laboratory in the U.S.A.. ${ }^{3-5}$ The colliding ions create the a plasma made of hot quarks, antiquarks and gluons, which are subjected to a short-lived strong magnetic field if the collisions are not central. ${ }^{6}$ Similar conditions may have also existed in the very early moments of our Universe. ${ }^{7}$

The chiral magnetic effect ${ }^{6,8-11}$ provides a particularly interesting example of the influence of the magnetic field on hot quark matter. Topological processes at the QCD scale may lead to a chiral asymmetry of quark matter which is characterized by a unequal densities of left and right quarks. The magnetic field may generate a dissipationless electric current in the parity-odd quark-gluon plasma leading to potentially observable phenomena in heavy-ion collisions. ${ }^{12-15}$

The hadron-scale magnetic field should also induce so-called magnetic catalysis, ${ }^{16-20}$ which implies, in particular, a steady enhancement of chiral symmetry breaking in the cold QCD vacuum as the external magnetic field strengthens. A strong magnetic field background makes a noticeable impact on the phase structure of the hot QCD vacuum which experiences, unexpectedly, an inverse magnetic catalysis: ${ }^{27,28}$ the transition temperature from the hadronic phase to the quark-gluon plasma phase turns out to be a slowly diminishing function of the background magnetic field. The latter phenomenon is not yet well understood theoretically. ${ }^{21-26}$

A strong magnetic field affects the phases of cold dense quark matter. ${ }^{29-33}$ The latter opens a possibility for experimental checks of the effects of a very strong magnetic field in the astrophysical setup because the strong magnetic background should affect certain macroscopic properties of the strongly magnetized neutron stars such as mass, adiabatic index, moment of inertia, and cooling curves. ${ }^{34}$

It was also suggested that the QCD vacuum may become an electromagnetic superconductor in a sufficiently strong magnetic field background at zerotemperature. ${ }^{35}$ The superconductivity of, basically, empty space, is mediated via spontaneous creation of a (charged) $\rho$-meson condensate if the magnetic field exceeds the critical value of $B_{c}^{\mathrm{QCD}} \sim 10^{16} \mathrm{~T}$. The ground state of the vacuum superconductor is characterized by an anisotropic and inhomogeneous geometric structure of the $\rho$-meson condensates ${ }^{36}$ which is suggested to possess intriguing metamaterial ("perfect lens") properties. ${ }^{37}$ The superconductivity of charged $\rho$ mesons may also be accompanied by a "tandem" superfluidity due to an emergent neutral $\rho$ - 
meson condensate. Although a first-principle treatment of this problem is impossible due to strong nonperturbative effects, the superconducting phase was shown to exist in various effective models of QCD such that effective bosonic $\rho$-meson electrodynamics, ${ }^{35,36}$ the effective fermionic Nambu-Jona-Lasinio model ${ }^{38,39}$ and in different holographic approaches. ${ }^{40-43}$ There are also signatures of the superconducting/superfluid phase in numerical simulations of lattice QCD. ${ }^{44,45}$

As the background magnetic field strengthens further, the electroweak sector of the Standard Model may also experience another superconducting, and, simultaneously, superfluid transition associated with a condensation of, respectively, the $W$ and $Z$ bosons at a critical magnetic field of the electroweak scale, $B^{\mathrm{EW}} \sim 10^{20} \mathrm{~T}$. The condensation of the $W$ bosons - first predicted by Ambjørn and Olesen in Refs. [46-48] - has a spatially inhomogeneous and anisotropic structure similarly to its QCD counterpart. The superconductivity and associated superfluidity of the $W$-condensed ground state was demonstrated in Ref. [54]. In even stronger magnetic fields the electroweak model may experience a second phase transition from the superconducting phase to a new electroweak phase in which the electroweak symmetry is completely restored. ${ }^{55}$

The key idea behind the magnetic-field-induced vacuum superconductivity is that the vacuum of charged vector particles (i.e., of the $W$ mesons) is unstable in the background of a sufficiently strong magnetic field provided these particles have an anomalously large gyromagnetic ratio $g_{m}=2$. The large value of $g_{m}$ guarantees that the magnetic moment of such particles is too large to withstand a spontaneous condensation at sufficiently strong external magnetic fields. The emergence of the electrically charged condensate implies, almost inevitably, electromagnetic superconductivity of the new vacuum ground state. Due to the anisotropic nature of the emergent superconductivity (the vacuum superconducts only along the axis of the magnetic field) the charged spin-1 condensate cannot screen the external magnetic field which induces the condensation and the Meissner effect is absent.

A related well-known example of the magnetic-field-induced instability of a trivial ground state is realized in QCD. A strong enough chromomagnetic field leads to an instability of the perturbative gluonic QCD vacuum since the gluon is a vector particle with the (color) gyromagnetic ratio $g=2 .{ }^{56}$ As a result of the instability, a spaghetti of chromomagnetic flux tubes is formed. These flux tubes tend to arrange themselves into a lattice structure similar to the Abrikosov lattice which is observed in the mixed state of a type-II superconductor subjected to a nearcritical external magnetic field. ${ }^{57,58}$ Other examples of the magnetic-field-induced condensation are discussed in the context of type-II superconductors, ${ }^{64-66}$ ferromagnetic superconductors ${ }^{49}$ and in nonperturbative holographic models of p-wave superconductivity. ${ }^{50-52}$

It is interesting to notice that the inhomogeneities of the magnetic-field-induced ground states are inevitably associated with the presence of topological vortexlike defects in the corresponding condensates. The condensates vanish in the center of the vortices while the phase of the condensate winds around the vortex core by a $2 \pi$ 
angle. In mean-field approaches the vortices are strictly parallel to the magnetic field axis while in the transverse plane the vortices form a regular lattice structure which is remarkably similar to an Abrikosov vortex lattice in a type-II superconductor. Thermal and quantum fluctuations may lead to instabilities of the regular vortex structure which may result in melting of the vortex lattice into a vortex liquid.

In this review we discuss magnetic-field-induced superconducting phenomena suggested to be realized in the vacuum of the Standard Model of particle interactions. In order to highlight differences and similarities between the suggested vacuum superconductivity and its solid-state counterpart, we briefly review in Section 1 certain basic features of the inhomogeneous ground states in the Ginzburg-Landau model of ordinary superconductivity. Sections 2 and 3 are devoted to the vacuum superconductivity in the bosonic sector of the electroweak model and in QCD, respectively. We discuss structure and symmetries of the inhomogeneous ground states, associated vortexlike topological defects, transports properties of the vacuum in the new phases as well as available lattice results.

\section{Vortex ground state in the Ginzburg-Landau model}

\subsection{Lagrangian and equations of motion}

Before going into details of superconducting ground states in field theories, it is instructive to discuss briefly certain general features of the phenomenological Ginzburg-Landau (GL) model of superconductivity. ${ }^{60}$ Since both Electroweak model and QCD are relativistic theories, we consider the relativistic version of the GL model:

$$
\begin{aligned}
\mathcal{L}(\phi, A) & =-\frac{1}{4} F_{\mu \nu} F^{\mu \nu}+\left(\mathfrak{D}_{\mu} \phi\right)^{*} \mathfrak{D}^{\mu} \phi-V(\phi), \\
V(\phi) & =-m^{2}\left|\phi^{2}\right|+\frac{\lambda}{4}|\phi|^{4} \equiv \frac{\lambda}{4}\left(|\phi|^{2}-\eta^{2}\right)^{2}+\mathrm{const},
\end{aligned}
$$

where $\mathfrak{D}_{\mu}=\partial_{\mu}-i e A_{\mu}$ is the covariant derivative and $A_{\mu}$ is the electromagnetic field with the strength $F_{\mu \nu}=\partial_{\mu} A_{\nu}-\partial_{\nu} A_{\mu}$. The superconducting order parameter $\phi \equiv|\phi| e^{i \varphi}$ is an electrically charged scalar field which plays the role of a scalar field of Cooper pairs. Without loss of generality, we assume that the boson field $\phi$ carries elementary electric charge $e$. The potential (2) is characterized by the mass parameter $m$ and the self-interaction coupling $\lambda$. The relativistic GL model (1), (2) is a renormalizable theory.

The superconducting state of the model (1) is characterized by a homogeneous condensate $|\langle\phi\rangle|=\eta$ with

$$
\eta=\sqrt{\frac{2 m^{2}}{\lambda}},
$$

and $m^{2}>0$. In this state the photon $A_{\mu}$ and the scalar excitation $\delta \phi=\phi-\langle\phi\rangle$ acquire, respectively, the following masses:

$$
m_{A}=\frac{2 e m}{\sqrt{\lambda}}, \quad m_{\phi}=\sqrt{2} m .
$$


The classical equations of motion of the GL model are

$$
\begin{aligned}
\mathfrak{D}_{\mu} \mathfrak{D}^{\mu} \phi+\frac{\lambda}{2}\left(|\phi|^{2}-\eta^{2}\right) \phi & =0, \\
\partial_{\nu} F^{\nu \mu}+J_{\mathrm{GL}}^{\mu} & =0,
\end{aligned}
$$

where

$$
J_{\mathrm{GL}}^{\mu}=-i e\left[\phi^{*} \mathfrak{D}^{\mu} \phi-\left(\mathfrak{D}^{\mu} \phi\right)^{*} \phi\right],
$$

is the electric superconducting current generated due to the presence of the Cooper pairs which are in turn described by the order parameter field $\phi$. The electric current is a conserved quantity, $\partial_{\mu} J_{\mathrm{GL}}^{\mu}=0$.

\subsection{Basic properties of an ordinary superconductor}

\subsubsection{First London equation: isotropic superconductivity}

Let us apply a very weak (test) external electromagnetic field to a superconducting ground state. Neglecting the effect of the external field on the uniform superconducting condensate $\phi$, one gets from Eq. (7):

$$
\partial^{\mu} J_{\mathrm{GL}}^{\nu}-\partial^{\nu} J_{\mathrm{GL}}^{\mu}=-m_{A}^{2} F^{\mu \nu}
$$

where the photon mass $m_{A}$ is given in Eq. (4).

Equation (8) describes a relativistic generalization of the two well-known London relations. For an electrically neutral superconductor $J_{0}(x) \equiv 0$, and the spacialtemporal component of Eq. (8) gives us the first London equation:

$$
\frac{\partial \vec{J}_{\mathrm{GL}}}{\partial t}=m_{A}^{2} \vec{E}
$$

where $E^{i} \equiv-F^{0 i}$ is the electric field. Equation (9) implies a linear (ballistic) growth of the electric current $\vec{J}_{\mathrm{GL}}$ along the direction of the external electric field $\vec{E}$, thus naturally indicating a vanishing electric resistance of the superconducting state.

Equation (9) describes homogeneous (independent of spatial coordinates) and isotropic (direction-independent) superconductivity. As we will see below, the magnetic-field-induced superconductivity of the vacuum in certain field theories is very different from the "classical" superconductivity: the vacuum superconductivity is both an inhomogeneous and anisotropic phenomenon.

\subsubsection{Second London Equation: the Meissner effect}

The spatial components of Eq. (8) give us the second London equation:

$$
\vec{\partial} \times \vec{J}_{\mathrm{GL}}=-m_{A}^{2} \vec{B} .
$$

Together with the Maxwell equation (6), $\vec{J}_{\mathrm{GL}}=\vec{\partial} \times \vec{B}$, the London relation (10) implies the following relation in the absence of the external electric field $(\vec{E}=0)$ :

$$
\left(-\Delta+m_{A}^{2}\right) \vec{B}=0 .
$$


This equation implies that the photon field acquires the mass $m_{A}$ given by Eq. (4) in the bulk of a superconductor. Consequently, the superconductor tends to expel the external magnetic field ("the Meissner effect"). Physically, the Meissner effect is realized because the external magnetic field induces the circulating superconducting currents (10) inside the superconductor. These currents, in turn, screen the external magnetic field since they induce their own magnetic field which is opposite to the external one.

Due to the Meissner effect, a weak magnetic field parallel to the surface boundary of the superconductor is always screened inside the bulk. The perpendicular magnetic field may either destroy the superconductivity or penetrate the superconductor and create a mixed "superconductor-normal state" phase of the Abrikosov vortices.

As we will see below, a magnetic-field-induced superconducting state in a field theory is described by an anisotropic version of the second London equation (10). As a consequence, the Meissner effect for the vacuum superconductivity cannot be formulated in a selfconsistent way. In a loose sense one may state that the Meissner effect is not realized in the magnetic-field induced superconducting state. As will be discussed below, a similar unusual phenomenon was suggested to happen in a type-II superconductor subjected to strong magnetic field. ${ }^{64-66}$

\subsubsection{Destructive role of magnetic field}

If the strength of external magnetic field $B_{\text {ext }}$ exceeds the critical value,

$$
B_{c 2}^{\mathrm{GL}}=\frac{m_{\phi}^{2}}{2 e} \equiv \frac{2 \lambda}{e} \eta^{2},
$$

then the superconducting state in an ordinary superconductor is completely destroyed in the bulk. Mathematically, this statement means that there are no solutions to the classical equations of motion (5) and (6) except for the trivial one $\phi=0$ if $B_{\text {ext }}>B_{c 2}^{\mathrm{GL}}$. On the contrary, we will show below that a strong enough magnetic field should induce and enhance the vacuum superconductivity in certain field theories instead of destroying it.

\subsubsection{Abrikosov lattice of vortices in the mixed state}

The classical equations of motion (5) and (6) admit a string-like topological solution which is known as the Abrikosov vortex. An elementary Abrikosov vortex carries a quantized total magnetic flux

$$
\int d^{2} x B(x)=\frac{2 \pi}{e},
$$

where the integration goes over the plane perpendicular to the vortex axis and $B(x) \equiv F_{12}(x)$ is the magnetic field along the third axis. In the very center of the vortex, $x=0$, the normal (nonsuperconducting) state is restored, $\phi(0)=0$. In the 
vicinity of the core of an elementary vortex, the scalar field has the following form:

$$
\phi\left(x_{\perp}\right) \propto\left|x_{\perp}\right| e^{i \varphi} \equiv x_{1}+i x_{2}
$$

where $\varphi$ is the azimuthal angle in the transverse plane, and $\left|x_{\perp}\right|=\sqrt{x_{1}^{2}+x_{2}^{2}}$ is the distance from the vortex center.

The vortex ground state may be realized in a type-II superconductor, which is characterized by the following relation between the model parameters:

$$
\lambda>e^{2} .
$$

Indeed, if the external magnetic field exceeds a certain (first) critical field, $B_{\text {ext }}>$ $B_{c 1}$, then it is energetically favorable for Abrikosov vortices to be formed in the superconducting material. Due to a repulsive interaction between the vortices in a type-II superconductor, the vortices may arrange themselves in a regular periodic structure called the Abrikosov lattice in order to minimize the net energy of the multi-vortex ensemble. Notice that the periodic lattice structure may not be absolutely stable since thermal and quantum fluctuations may disorder the vortex lattice by melting it into various forms vortex liquids or vortex glasses. ${ }^{61}$

In the simplest version of the vortex phase diagram, the vortex phases are formed in a background of magnetic field with the strength restricted by the critical values, $B_{c 1}<B<B_{c 2}$, which depend on temperature and other parameters. The background magnetic field penetrates the superconductor in the form of the vortices, thus partially restoring the normal phase in the vortex cores. Therefore the vortex phase is often called the "mixed phase". In the next section we discuss properties of this phase in more details.

\subsection{Vortex lattices}

The vortex lattices are solutions of classical equations of motion (5) and (6) in the presence of the magnetic field background. Since this system of equations is independent of the time $x_{0}$ and longitudinal space $x_{3}$ coordinates, one can choose the ground state to be a function of the transverse coordinates $x_{1}$ and $x_{2}$ only. Then, it is convenient to use the complex notation both for the transverse coordinates in two spatial dimensions,

$$
z=x_{1}+i x_{2}, \quad \bar{z}=x_{1}-i x_{2},
$$

and for the transverse components of the vectors $\mathcal{O}_{\mu}=\partial_{\mu}, A_{\mu}$, etc:

$$
\mathcal{O}=\mathcal{O}_{1}+i \mathcal{O}_{2}, \quad \overline{\mathcal{O}}=\mathcal{O}_{1}-i \mathcal{O}_{2}, \quad \mathcal{O}_{12}=-\frac{i}{2}(\bar{\partial} \mathcal{O}-\partial \overline{\mathcal{O}})
$$

The last formula corresponds to the real-valued field strength of a gauge vector field $\mathcal{O}_{\mu}$.

In this review we will always consider a uniform static magnetic field which is parallel to the $x_{3}$ axis, $B_{\text {ext }, i}=B_{\text {ext }} \delta_{i 3}$. It is also convenient to describe the background gauge field in a "symmetric" gauge given by the following electromagnetic 
potential,

$$
A_{1}^{\mathrm{ext}}=-\frac{B_{\mathrm{ext}}}{2} x_{2}, \quad A_{2}^{\mathrm{ext}}=\frac{B_{\mathrm{ext}}}{2} x_{1}, \quad A_{3}^{\mathrm{ext}}=A_{0}^{\mathrm{ext}}=0 .
$$

We will ignore quantum fluctuations of the gauge and scalar fields, thus treating the problem at a classical level. Finally, without loss of generality, we will always assume that $e B_{\text {ext }}>0$.

The equations of motion (5) and (6) can now be rewritten in the complexified form:

$$
\begin{aligned}
\left(\mathfrak{D} \overline{\mathfrak{D}}+m^{2}-e B\right) \phi-\lambda|\phi|^{2} \phi & =0, \\
\partial\left(B+e|\phi|^{2}\right)-2 e \phi^{\dagger} \mathfrak{D} \phi & =0,
\end{aligned}
$$

where the covariant derivatives are as follows:

$$
\mathfrak{D}=\partial+\frac{e}{2} B_{\text {ext }} z, \quad \overline{\mathfrak{D}}=\bar{\partial}+\frac{e}{2} B_{\text {ext }} \bar{z} .
$$

In order to characterize the vortex phase, it is convenient to consider a vicinity of the transition region close to the upper critical field $B_{c 2}$. In this case the uniform time-independent magnetic field $B_{\text {ext }}$ is chosen to be slightly smaller than the critical value (12), so that $B_{\text {ext }}<B_{c}^{\mathrm{GL}}$ and $\left|B_{\text {ext }}-B_{c}^{\mathrm{GL}}\right| \ll B_{c}^{\mathrm{GL}}$. Then the superconducting condensate can be treated as a small quantity, $|\langle\phi\rangle| \ll \eta \equiv\left|\langle\phi\rangle\left(B_{\text {ext }}=0\right)\right|$, and consequently, the classical equations of motion (19) and (20) can be linearized. In particular, linearized Eq. (19) along with the requirement for the solution to have a finite energy density, lead to the following equation for the superconducting condensate $\phi$ :

$$
\overline{\mathfrak{D}} \phi=0 .
$$

Following the original work of Abrikosov, ${ }^{62}$ it is convenient to chose a general solution of Eq. (22) in a form of the sum,

$$
\phi(z)=\sum_{n \in \mathbb{Z}} C_{n} h_{n}\left(\nu, \frac{z}{L_{B}}, \frac{\bar{z}}{L_{B}}\right),
$$

over the lowest Landau levels

$$
h_{n}(\nu, z, \bar{z})=\exp \left\{-\frac{\pi}{2}\left(|z|^{2}+\bar{z}^{2}\right)-\pi \nu^{2} n^{2}+2 \pi \nu n \bar{z}\right\} .
$$

Here the quantity

$$
L_{B}=\sqrt{\frac{2 \pi}{e B}},
$$

is the magnetic length and the arbitrary complex $C_{n}$ and real $\nu$ parameters parametrize the ground state solution (23). It is the parameters $C_{n}$ that determine a particular geometric form of the spatial inhomogeneities of the solution (23). As we will see below, the solution (23) describes a general ensemble of vortices with singularities of the form (14) distributed in the transversal plane. Regular periodic solutions correspond to vortex lattices. 
As we look for an inhomogeneous solution corresponding to a periodic lattice structure, we fix the variable $n$ in Eq. (22) to take integer values, $n \in \mathbb{Z}$. In order to ensure a regular structure of the lattice, we choose the coefficients $C_{n}$ in a periodic manner:

$$
C_{n+N}=C_{n}
$$

The solution with $N=1$ (with $C_{n}=C_{0}$ for all $n$ ) corresponds to a square vortex lattice. This solution was first found in the original Abrikosov paper. ${ }^{62}$

In order to determine the ground state values of the vortex lattice parameters $\left(N, C_{n}\right.$ and $\left.\nu\right)$ one should first minimize the energy of the solution for a fixed value of the periodicity parameter $N$, and then choose the optimal value of $N$ which corresponds to the global energy minimum. Since the action density is a function of the transversal coordinates $x_{1}$ and $x_{2}$, it is convenient to consider the energy density which is averaged over the transversal plane:

$$
\langle\mathcal{E}\rangle=\frac{1}{2} B_{\text {ext }}^{2}-\left(m^{2}-e B_{\text {ext }}\right)\left\langle|\phi|^{2}\right\rangle_{\perp}+\frac{e^{2}}{2}\left\langle|\phi|^{2}\right\rangle_{\perp}^{2}+\frac{1}{2}\left(\lambda-e^{2}\right)\left\langle|\phi|^{4}\right\rangle_{\perp} .
$$

The brackets $\langle\cdots\rangle_{\perp}$ indicate a mean value in the transversal $\left(x_{1}, x_{2}\right)$ plane:

$$
\langle\mathcal{O}\rangle_{\perp}=\frac{1}{\text { Area }_{\perp}} \int d x_{1} \int d x_{2} \mathcal{O}\left(x_{1}, x_{2}\right)
$$

where $A a_{\perp}$ is the area of the transversal plane.

Using the explicit form of the solution (23) and (24), one can show that

$$
\begin{aligned}
\left\langle|\phi|^{2}\right\rangle_{\perp}= & \frac{1}{\sqrt{2}|\nu|} \lim _{M \rightarrow \infty} \frac{1}{M} \sum_{n=-M / 2}^{M / 2}\left|C_{n}\right|^{2} \\
\left\langle|\phi|^{4}\right\rangle_{\perp}= & \frac{1}{2|\nu|} \lim _{M \rightarrow \infty} \frac{1}{M} \sum_{n_{1}=-M / 2}^{M / 2} \sum_{n_{2} \in \mathbb{Z}} \sum_{n_{3} \in \mathbb{Z}} e^{-\pi \nu^{2}\left(n_{2}^{2}+n_{3}^{2}\right)} \\
& \times C_{n_{1}+n_{2}} C_{n_{1}}^{*} C_{n_{1}+n_{3}} C_{n_{1}+n_{2}+n_{3}}^{*} .
\end{aligned}
$$

In the case of the $N$-fold symmetry (26) the above equations get simplified:

$$
\begin{aligned}
\left\langle|\phi|^{2}\right\rangle_{\perp} & =\frac{1}{\sqrt{2} N|\nu|} \sum_{n=0}^{N-1}\left|C_{n}\right|^{2} \\
\left\langle|\phi|^{4}\right\rangle_{\perp} & =\frac{1}{2 N|\nu|} \sum_{n_{1}=0}^{N-1} \sum_{n_{2} \in \mathbb{Z}} \sum_{n_{3} \in \mathbb{Z}} e^{-\pi \nu^{2}\left(n_{2}^{2}+n_{3}^{2}\right)} C_{n_{1}+n_{2}} C_{n_{1}}^{*} C_{n_{1}+n_{3}} C_{n_{1}+n_{2}+n_{3}}^{*}
\end{aligned}
$$

In a special case of the Bogomolny limit, ${ }^{63}$

$$
\lambda=e^{2},
$$

the energy (27) depends only on the spatial average of the condensate squared $\left\langle|\phi|^{2}\right\rangle$ given by the sum of the squares of the coefficients $C_{n}$, Eq. (31). In this specific limit 
an infinite amount of the vortex structures correspond to the same minimum of the energy functional

$$
\mathcal{E}_{\min }^{\lambda=e^{2}}=\frac{1}{2}\left(2 B_{\text {ext }}-B_{c}\right) B_{c}
$$

because the energy minimum is only fixed by the specific value of the superconducting condensate,

$$
\left\langle|\phi|^{2}\right\rangle_{\perp}^{\lambda=e^{2}}=\frac{1}{e}\left(B_{c}-B_{\text {ext }}\right) .
$$

The mean value of the condensate (35) defines a mean value of the squared coefficients $C_{n}$ while the precise values of these coefficients remain undetermined. Thus, in the Bogomolny limit the vortex ground state need not be a regular vortex lattice since the parameters $C_{n}$ fix a geometric form of the spatial inhomogeneities of the solution (23). This observation nicely fits the fact that parallel Abrikosov vortices do not interact in the Bogomolny limit, so that a periodic lattice structure is unlikely to be formed. In the type-II regime (15) the vortices repel each other so that the regular (periodic) vortex lattice is a preferred structure of the ground state in this case.

Since we are working with a type-II superconductor (15), the last term in the energy density is always positive (27). Therefore the global minimum of the energy (27) corresponds to a global minimum of a dimensionless quantity,

$$
\beta_{A}=\frac{\left\langle|\phi|^{4}\right\rangle_{\perp}}{\left\langle|\phi|^{2}\right\rangle_{\perp}^{2}},
$$

which is called the Abrikosov ratio. ${ }^{62}$ According to Eqs. (31) and (32) the Abrikosov ratio (36) can be expressed via the parameters of the ground state solution (23). In the GL model the Abrikosov ratio (36) of the ground state is independent of the value of the external magnetic field $B_{\text {ext }}$.

For the simplest lattice of the square type, $N=1$, the Abrikosov ratio (36) is $\beta(N=1) \approx 1.180$ which is reached at $\nu=1$. However, at $N=2$ the Abrikosov ratio (and, consequently, the energy density) is even smaller, $\beta(N=2) \approx 1.1596$, which implies that the ground state corresponds to the following parameters:

$$
N=2: \quad C_{1}= \pm i C_{0}, \quad \nu=\frac{\sqrt[4]{3}}{\sqrt{2}} \approx 0.9306
$$

This minimal-energy periodic pattern describes an equilateral triangular lattice (which is sometimes called "hexagonal" lattice). At higher odd values of $N$, the Abrikosov ratio is higher than the $N=2$ minimum (for example, $\beta_{N=3}=1.167$ ) while at even values of $N$ the minimization converges to the two-fold pattern corresponding to the triangular lattice $\left(\beta_{N=2 k}=\beta_{N=2}\right.$ with $\left.k \in \mathbb{Z}\right)$. A nice review of the vortex lattice structures in the GL theory of type-II superconductors can be found in Ref. [61].

Examples of square $(N=1)$ and triangular $(N=2)$ Abrikosov vortex lattices are shown in Fig. 1. The darker pointlike regions indicate the positions of the vortex 

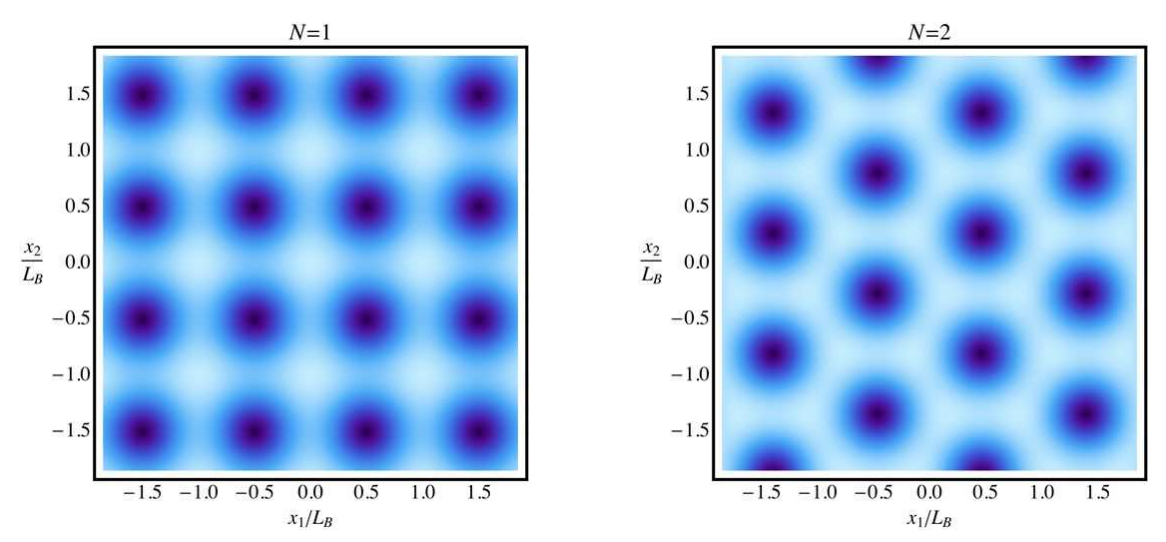

Fig. 1. The density plot of the superconducting condensate in the transverse $\left(x_{1}, x_{2}\right)$ plane for $N=1$ (left) and $N=2$ (right) vortex lattice solutions in the GL model.

cores where the condensate $\phi=\phi\left(x_{1}, x_{2}\right)$ takes lower values. The condensate vanishes at the center of each vortex, $\phi=0$, while the phase of the scalar field winds around a geometrical center of each vortex, $\arg \phi=\varphi$, where $\varphi$ is a two-dimensional azimuthal angle of a coordinate system centered at the vortex's origin (14).

The solution $\phi=\phi(z)$ of the Abrikosov equation (22) determines the behavior of the magnetic field inside the superconductor,

$$
B(z)=B_{\text {ext }}+e\left[|\phi(z)|^{2}-\left\langle|\phi|^{2}\right\rangle_{\perp}\right]
$$

which is a certain function of the transversal coordinates $x_{1}$ and $x_{2}$. An additive coordinate-independent term $\left\langle|\phi|^{2}\right\rangle_{\perp}$ in the solution (38) is a normalization term which imposes conservation of the magnetic flux coming through the transversal plane,

$$
\langle B\rangle_{\perp}=B_{\text {ext }}
$$

According to Eq. (38), the magnetic field is concentrated at the positions of the vortices, so that the distribution of the magnetic field is visually similar to Fig. $1 .{ }^{61}$

\subsection{Reentrant superconductivity in strong magnetic field}

Summarizing, the ordinary superconductivity is usually suppressed by magnetic field. In type-II superconductors the increasing magnetic field leads to appearance of a mixed state of the Abrikosov vortices if the strength of the field exceeds the first critical field, $B_{c 1}$. In the mixed phase the superconducting state coexist with the normal state which is restored in the cores of the vortices. As the strength of the magnetic field increases further, the condensate of the Cooper pairs gets smaller and it eventually disappears when the second critical magnetic field $B_{c 2}$ is reached. The analysis of the GL model shows that above $B_{c 2}$ a normal, nonsuperconducting phase is restored.

However, there are indications in certain theoretical approaches beyond the simplest $s$-wave GL model that a superconducting regime may exist even above the sec- 
ond critical magnetic field $B_{c 2}$. It was suggested in Ref. [64-66] that in a very strong magnetic field the Abrikosov flux lattice of a type-II superconductor may enter a quantum limit of the low Landau level dominance, characterized by a spin-triplet pairing, absence of the Meissner effect, and a superconducting flow along the magnetic field axis. This kind of superconductivity turns out to be highly anisotropic and inhomogeneous. The mentioned quantum limit is reached when the magnetic length $1 / \sqrt{|e B|}$ becomes of the order of the correlation length $\xi$. Due to the spintriplet pairing the induced superconductivity cannot be described by a scalar order parameter $\phi$ used in the simplest version of the GL model (5).

In solid state physics the magnetic-field-induced anisotropic superconductivity is sometimes called the "reentrant" superconductivity because the system should normally "exit" a superconducting state as an increasing external magnetic field suppresses superconductivity via diamagnetic and Pauli pair breaking effects. Although it is unclear whether this particular mechanism of the reentrant superconductivity works in real superconductors, the magnetic-field-induced restoration of superconductivity was experimentally observed in certain materials like an uranium superconductor URhGe. ${ }^{67,68}$

In the next Sections we discuss the phenomenon of the magnetic-field-induced superconductivity in certain field theories at zero temperature. This effect has all basic features of the reentrant superconductivity in type-II superconductors: ${ }^{64-66}$ superconductivity is mediated by certain electrically charged condensates of spinone particles, the vacuum superconductor exhibits no Meissner effect while the vacuum superconductivity is suggested to be highly anisotropic. Below we discuss this phenomenon in the Electroweak model (Section 2) where the condensed electrically charged spin-1 states correspond to the $\mathrm{W}$ bosons and then we review the current situation in QCD (Section 3) in which the role of the Cooper pairs is suggested to be played by composite quark-antiquark states with quantum numbers of $\rho$ mesons.

\section{Electroweak model in strong magnetic field}

\subsection{Structure of the ground state}

\subsubsection{Lagrangian}

The bosonic sector of the Electroweak model is described by the following Lagrangian,

$$
\mathcal{L}=-\frac{1}{4} W_{\mu \nu}^{a} W^{a, \mu \nu}-\frac{1}{4} X_{\mu \nu} X^{\mu \nu}+\left(D_{\mu} \Phi\right)^{\dagger}\left(D^{\mu} \Phi\right)-\lambda\left(|\Phi|^{2}-\frac{v^{2}}{2}\right)^{2},
$$

where $\Phi \equiv\left(\phi_{1}, \phi_{2}\right)^{T}$ is the scalar complex Higgs doublet, and $W_{\mu}^{a}$ and $X_{\mu}$ are the $S U(2)_{L}$ and $U(1)_{X}$ gauge fields, respectively. The Higgs field interacts with the vector gauge fields via the covariant derivative

$$
D_{\mu}=\partial_{\mu}-\frac{i g}{2} \tau^{a} W_{\mu}^{a}-\frac{i g^{\prime}}{2} X_{\mu}
$$


where $g$ and $g^{\prime}$ are the coupling constants associated with the $S U(2)_{L}$ and $U(1)_{X}$ gauge groups, respectively, and $\tau^{a}$ are the Pauli matrices. The field strengths of the $S U(2)_{L}$ and $U(1)_{X}$ gauge fields are, respectively, as follows:

$$
\begin{aligned}
W_{\mu \nu}^{a} & =\partial_{\mu} W_{\nu}^{a}-\partial_{\nu} W_{\mu}^{a}+g \epsilon^{a b c} W_{\mu}^{b} W_{\nu}^{c}, \\
X_{\mu \nu} & =\partial_{\mu} X_{\nu}-\partial_{\nu} X_{\mu} .
\end{aligned}
$$

In the ground state the Higgs field $\Phi$ acquires a vacuum expectation value, $\langle\Phi\rangle \neq 0$, and the electroweak symmetry gets spontaneously broken down to its electromagnetic subgroup,

$$
S U(2)_{L} \times U(1)_{X} \rightarrow U(1)_{\mathrm{em}}
$$

Since three out of four generators of the gauge group are broken (44), the theory contains three massive vector bosons $\left(W^{ \pm}\right.$and $Z$ ) as well as one massless electromagnetic gauge field $A_{\mu}$ associated with the unbroken subgroup. The massive $Z_{\mu}$ boson and the massless electromagnetic field $A_{\mu}$ appear - in the unitary gauge with $\langle\Phi\rangle=(0, v)^{T}$ - as a combination of the third component of the non-Abelian gauge field $W_{\mu}^{3}$ and the Abelian gauge field $X_{\mu}$ :

$$
\begin{aligned}
W_{\mu}^{3} & =\sin \theta A_{\mu}+\cos \theta Z_{\mu}, \\
X_{\mu} & =\cos \theta A_{\mu}-\sin \theta Z_{\mu},
\end{aligned}
$$

where $\theta$ is the electroweak (Weinberg) angle and $e=g \sin \theta=g^{\prime} \cos \theta$ is the elementary electric charge $e=|e|$. The masses of the $W$ boson, $Z$ and Higgs bosons are, respectively, as follows:

$$
M_{W}=\frac{g v}{2}, \quad M_{Z}=\frac{g v}{2 \cos \theta}, \quad M_{H}=\sqrt{2 \lambda} v .
$$

\subsubsection{Instability of the ground state in background magnetic field}

The instability of the electroweak vacuum in a sufficiently strong classical magnetic field was first demonstrated by Ambjørn and Olesen. ${ }^{46-48}$ Their arguments can briefly be summarized as follows.

Let us consider a uniform static magnetic field along the $x_{3}$ axis. In the presence of the magnetic field background the transverse $(\mu=1,2)$ components of the $W$ boson field give the following contribution to the mass term of the quadratic part of the electroweak Lagrangian (40):

$$
\delta \mathcal{L}_{W_{\perp}}^{(2)}=\left(W_{1}^{\dagger}, W_{2}^{\dagger}\right)\left(\begin{array}{cc}
M_{W}^{2} & -i e B_{\mathrm{ext}} \\
i e B_{\mathrm{ext}} & M_{W}^{2}
\end{array}\right)\left(\begin{array}{l}
W_{1} \\
W_{2}
\end{array}\right) .
$$

The eigenvalues of the mass matrix in Eq. (48),

$$
\mu_{ \pm}^{2}=M_{W}^{2} \pm e B_{\text {ext }},
$$

indicate that one of the masses, $\mu_{-}$, vanishes at the critical magnetic field

$$
B_{c 1}^{\mathrm{EW}}=\frac{M_{W}^{2}}{e} \simeq 1.1 \times 10^{20} \mathrm{~T}
$$


where $M_{W}=80.4 \mathrm{GeV}$ is the mass of the $W$ boson. The mass eigenvalue $\mu_{-}$becomes purely imaginary at $B>B_{c 1}^{\mathrm{EW}}$, thus signaling a tachyonic instability of the $\left\langle W_{\mu}\right\rangle=0$ ground state towards condensation of the transverse components of the $W$ field. The runaway of the $W$ field is stabilized at a nonzero value of $\left\langle W_{\mu}\right\rangle$ by higher-order interactions which are not present in the mass term of the quadratic Lagrangian (48).

The eigenvectors $w_{ \pm}$corresponding to $\mu_{ \pm}$eigenvalues have the following form in the transverse $\left(x_{1}, x_{2}\right)$ space:

$$
w_{+}=\left(\begin{array}{l}
W_{1} \\
W_{2}
\end{array}\right)_{+}=\frac{\bar{W}}{2}\left(\begin{array}{l}
1 \\
i
\end{array}\right), \quad w_{-}=\left(\begin{array}{l}
W_{1} \\
W_{2}
\end{array}\right)_{-}=\frac{W}{2}\left(\begin{array}{c}
1 \\
-i
\end{array}\right),
$$

where $W$ and $\bar{W}$ are scalar fields:

$$
W=W_{1}+i W_{2}, \quad \bar{W}=W_{1}-i W_{2} .
$$

It is worth stressing that the mass terms of the $Z$-boson field and the longitudinal components $W_{3}$ and $W_{0}$ of the charged $W$-boson field are not affected by the magnetic field at the classical level.

The tachyonic instability of the $W$ boson at $B_{\text {ext }}>B_{c 1}^{\mathrm{EW}}$ indicates that the $B_{\text {ext }}=0$ ground state is no more stable at $B_{\text {ext }}>B_{c 1}$. As we will see below, the new ground state is characterized by the presence of the charged $W$-boson condensate which leads to anisotropic superconductivity. ${ }^{54}$

\subsubsection{Equations of motion and energy density}

The classical equations of motion of the electroweak theory (40) are as follows:

$$
\begin{aligned}
& 0=\partial^{\mu} W_{\mu \nu}^{a}+g \epsilon^{a b c} W^{b \mu} W_{\mu \nu}^{c}-\frac{i g}{2}\left[\left(D_{\mu} \Phi\right)^{\dagger} \tau^{a} \Phi-h . c .\right] \\
& \left.0=\partial^{\mu} X_{\mu \nu}-\frac{i g^{\prime}}{2}\left(D_{\mu} \Phi\right)^{\dagger} \Phi-h . c .\right) \\
& 0=-D_{\mu} D^{\mu} \Phi+2 \lambda \Phi\left(|\Phi|^{2}-\frac{v^{2}}{2}\right) .
\end{aligned}
$$

Since the system of equations (18), (45), (46) and (53) is invariant with respect to translations along the $x_{3}$ and $x_{0}$ axes it is natural to assume that the ground state of the system is described by condensates which are independent of the longitudinal $x_{3}$ and time $x_{0}$ coordinates. Thus, following our experience with the Ginzburg-Landau model we will search for two dimensional solutions of Eq. (53).

The conventions (17) for the complex transversal coordinates $x_{1}$ and $x_{2}$ are consistent with the definition of the scalar fields $W$ and $\bar{W}$ given in (52). The $W$ field in Eq. (52) corresponds to the $\mu_{-}$eigenvalue (49) which experiences a tachyonic instability. Thus, the field combination $W$ should be condensed if the magnetic field exceeds the critical value $(50)$ so that $W=W\left(x_{1}, x_{2}\right) \neq 0$. On the other hand, the $\bar{W}$ component corresponds to the $\mu_{+}$eigenvalue which is always positive. Therefore we can put $\bar{W} \equiv 0$ in our analysis. As we discuss later, the strong magnetic field 
background should also affect the expectation value of the Higgs field and the neutral gauge boson $Z$.

It is convenient to fix the unitary gauge with respect to the non-Abelian $S U(2)$ subgroup of the electroweak gauge group, $\Phi=(0, \phi)^{T}$ and consider $\phi=\phi\left(x_{1}, x_{2}\right)$ as a real-valued field to be determined by the equations of motion. Then, the complexified equations of motion (53) take the following form:

$$
\begin{aligned}
\mathfrak{D} \overline{\mathfrak{D}} W & =\left(\frac{g^{2}}{2}|W|^{2}-g \cos \theta Z_{12}-e F_{12}+\frac{g^{2}}{2} \phi^{2}\right) W, \\
\overline{\mathfrak{D}}^{2} W & =0 \\
\bar{\partial} F_{12} & =\frac{e}{2} \bar{\partial}|W|^{2}+\frac{e}{2} W^{\dagger} \overline{\mathfrak{D}} W \\
\partial \bar{\partial} \phi & =\frac{g^{2}}{4 \cos ^{2} \theta}|Z|^{2} \phi+\frac{g^{2}}{4}|W|^{2} \phi+2 \lambda \phi\left(\phi^{2}-\frac{v^{2}}{2}\right), \\
0 & =\cos \theta \bar{\partial} F_{12}-\sin \theta \bar{\partial} Z_{12}+i g^{2} \frac{\sin \theta}{2 \cos ^{2} \theta} \bar{Z} \phi^{2} \\
0 & =\phi \overline{\mathfrak{D}} W+2 W\left(\bar{\partial}+i \frac{g}{2 \cos \theta} \bar{Z}\right) \phi,
\end{aligned}
$$

where $\mathfrak{D}=\mathcal{D}+i g \cos \theta Z$ is a covariant derivative.

The analysis of the quadratic action (48) suggests that the condensation of the $W$ bosons at high magnetic field, $B>B_{c 1}^{\mathrm{EW}}$, lowers the energy of the ground state, $E=E\left(x_{1}, x_{2}\right)$. The local energy density is given by the $T^{00}$ component of the energy momentum tensor $T^{\mu \nu}$,

$$
\begin{gathered}
E\left(x_{1}, x_{2}\right)=\frac{1}{2}|(\overline{\mathcal{D}}+i g \cos \theta \bar{Z}) W|^{2}+\frac{1}{2} Z_{12}^{2}+\frac{1}{2} B^{2}+\frac{g^{2}}{8}|W|^{4}+\bar{\partial} \phi \partial \phi \\
+\frac{1}{2}\left(-e B-g \cos \theta Z_{12}+\frac{g^{2}}{2} \phi^{2}\right)|W|^{2}+\frac{g^{2}}{4 \cos ^{2} \theta}|Z|^{2} \phi^{2}+\lambda\left(\phi^{2}-\frac{v^{2}}{2}\right)^{2},
\end{gathered}
$$

where we have used the complex notations (17) for the transverse coordinates and fields.

The classical equations of motion were first analyzed ${ }^{46-48}$ in the Bogomolny limit, $M_{Z}=M_{H}$, where $M_{H}$ and $M_{Z}$ are the masses of the Higgs and $Z$ bosons, respectively (47). In the Bogomolny limit the second-order differential equations of motion (53) may be reduced to differential equations of the first order, simplifying the problem drastically. Following Ref. [53] we do not restrict ourselves to the Bogomolny limit in our analysis below.

\subsubsection{Phase structure in strong magnetic field: a brief overview}

Besides the phase transition at the critical magnetic field (50) the electroweak model at zero temperature is suggested to have a second critical field: ${ }^{69,70}$

$$
B_{c 2}^{\mathrm{EW}}=\frac{M_{H}^{2}}{e} \simeq 2.4 \times 10^{20} \mathrm{~T},
$$

where $M_{H}=125 \mathrm{GeV}$ is the mass of the Higgs boson. ${ }^{71,72}$ 
At the first critical magnetic field $B=B_{c 1}^{\mathrm{EW}}$ the vacuum turns from the standard insulating phase into the superconducting phase which is characterized by the presence of charged and neutral vector condensates, $\langle W\rangle \neq 0$ and $\langle Z\rangle \neq 0$. In both insulating $\left(B<B_{c 1}^{\mathrm{EW}}\right)$ and superconducting $\left(B_{c 1}^{\mathrm{EW}}<B<B_{c 2}^{\mathrm{EW}}\right)$ phases the Higgs condensate is nonzero, $\langle\Phi\rangle \neq 0$, so that the electroweak symmetry is spontaneously broken.

As the background magnetic field strengthens further, the electroweak sector of the standard model experiences a second phase transition associated with the restoration of the electroweak symmetry at $B=B_{c 2}^{\mathrm{EW}}$. In the third, symmetric phase the Higgs condensate is zero, $\langle\Phi\rangle=0$.

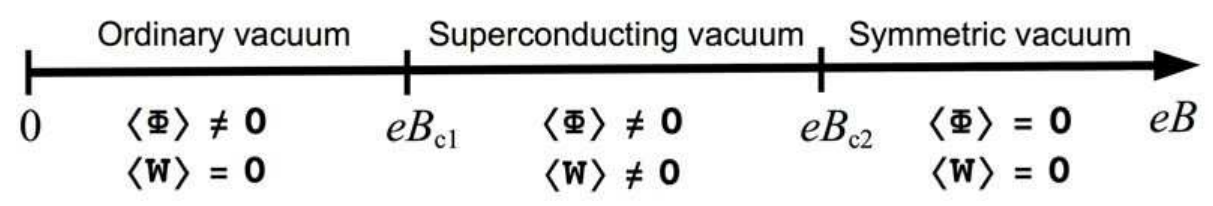

Fig. 2. The phase diagram of the electroweak model in a background magnetic field.

Thus, the phase diagram of Electroweak model in strong magnetic field contains three phases ${ }^{55,69,70}$ which are separated by two critical magnetic fields given in Eqs. (50) and (61). The phase diagram is illustrated in Fig. 2. Below we discuss the phase structure of the electroweak model in more details.

\subsection{Superconducting vacuum near the first critical magnetic field}

The mean-field analysis indicates that the magnetic-field-induced transition at the first critical magnetic field is of the second order. ${ }^{46-48,53}$ This fact implies, in particular, that the condensate of the $W$ bosons is a continuous function of the magnetic field $B_{\text {ext }}$. As a consequence, in the vicinity of the first critical magnetic field (50), $B_{\text {ext }} \geqslant B_{c 1}^{\mathrm{EW}}$ with $\left|B_{\text {ext }}-B_{c 1}^{\mathrm{EW}}\right| \ll B_{c 1}^{\mathrm{EW}}$, the $W$ condensate should be sufficiently small,

$$
\epsilon=\frac{|W|}{M_{W}} \ll 1,
$$

so that the equations of motion (54)-(59) may be linearized and the analysis of the superconducting phase may be simplified drastically. In this section we discuss the structure of the superconducting phase at $B<B_{c 2}^{\mathrm{EW}}$ in the vicinity of the first critical magnetic field for arbitrary Higgs masses.

\subsection{1. $W$ condensate and energy density}

A combination of the first two linearized equations of motion, (54) and (55), along with the requirement of minimization of the energy density (60), gives us a simple 
Abrikosov equation, ${ }^{46}$

$$
\overline{\mathcal{D}} W \approx \overline{\mathfrak{D}} W=0
$$

where the covariant derivative is given by Eq. (21). Equation (63) is valid up to $O\left(\epsilon^{2}\right)$ corrections where the smallness parameter $\epsilon$ is defined in Eq. (62).

As we have discussed earlier in Section 1.3, the Abrikosov equation (63) appears in the GL model of the ordinary superconductivity ${ }^{61}$ where it describes an inhomogeneous ground state of a type-II superconductor in a magnetic field background. Thus, having encountered Eq. (63) in the electroweak theory, one may naturally suggest that the ground state of electroweak vacuum in a strong magnetic field is described by an ensemble of vortices embedded in the $W$ condensate.

Following Abrikosov, ${ }^{62}$ a general solution of Eq. (63) in the symmetric gauge (18) can be written similarly to the vortex solution in the Ginzburg-Landau model (23):

$$
W(z)=\sum_{n \in \mathbb{Z}} C_{n} h_{n}\left(\nu, \frac{z}{L_{B}}, \frac{\bar{z}}{L_{B}}\right),
$$

where $L_{B}$ is the magnetic length (25). The solution (64) is parameterized by arbitrary complex-valued parameters $C_{n}$ and a real-valued parameter $\nu$. The sum in Eq. (64) is an expansion of the ground state wave-function $W(z)$ over the lowest Landau levels $h_{n}$, Eq. (24), of the charged $W$ field in the background of the external magnetic field.

To ensure the regular structure of the ground state, the complex coefficients $C_{n}$ are chosen in the $N$-periodic manner (26) similarly to the case of the GL model. A solution with $N=1$ and $\nu=1$ corresponds to a square pattern of the vortex lattice. As it happens in the GL model, the square-lattice solution represents a local, rather than global, minimum of the energy functional.

The global energy minimum is reached for the equilateral triangular lattice with the familiar parameters ${ }^{46-48,53}(37)$. All coefficients $C_{n}$ are expressed via the single proportionality factor $C_{0}$ which can be found numerically by a minimization of the energy density (60) at fixed values of the background magnetic field $B_{\text {ext }}$ and a fixed Higgs mass $M_{H}$. This procedure allows us to determine the $W$ condensate (64), the energy density (60) and other interesting quantities.

The numerical minimization of the mean energy density (60) was performed in Ref. [54] (see also Ref. [53]). As one can expect from the analysis of the quadratic action, the condensation of the $W$ bosons makes the energy density smaller compared to its value in the trivial ground state provided the magnetic field exceeds its critical value, $B \geqslant B_{c}^{\mathrm{EW}}$. The dependence of the mean (i.e., averaged in the transverse plane) $W$ condensate on the strength of the magnetic field $B$ is shown in Fig. 3(left) at various values of the Higgs mass including the actual physical mass of the Higgs boson. ${ }^{71,72}$ The $W$-boson condensation is an energetically favorable state in the strong magnetic field background, because the condensation energy, which corresponds to the difference in energy between the condensed and non-condensed 

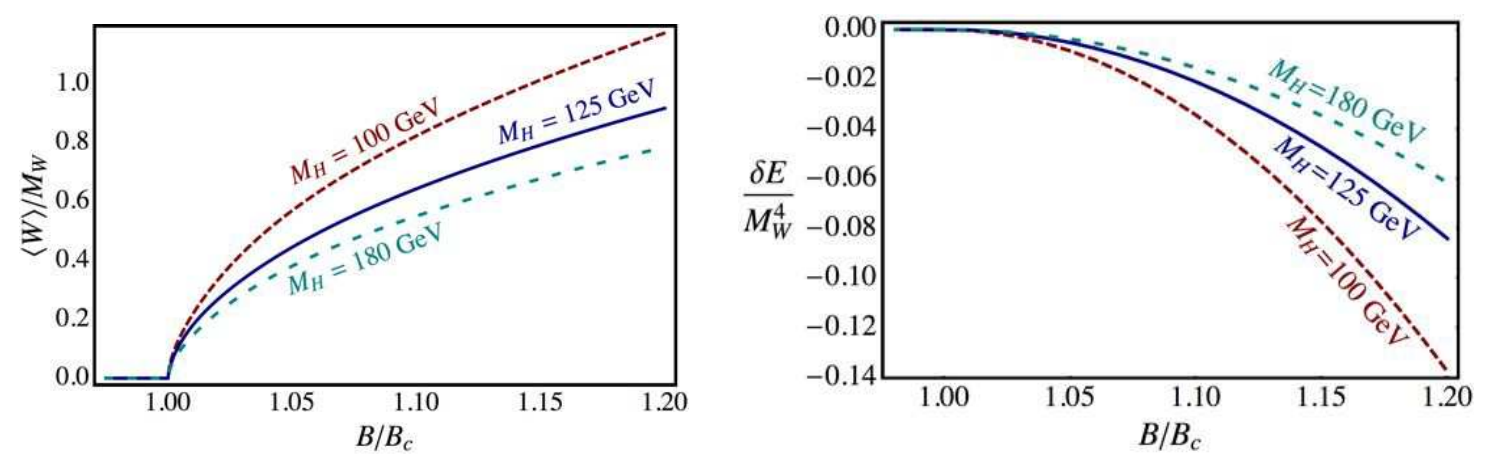

Fig. 3. (Left) The cell-averaged $W$ condensate $\left\langle|W|^{2}\right\rangle^{1 / 2}$ and (right) the condensation energy density (60), $\delta E$ vs. the strength of the magnetic field $B$ in units of the critical magnetic field $B \equiv B_{c}^{\mathrm{EW}}$. The plots are given for various Higgs masses $M_{H}$ including the physical value of the Higgs mass ${ }^{71,72}$ shown by the solid lines.

states,

$$
\delta E=\langle E\rangle_{W}-\langle E\rangle_{W=0}
$$

is a negative quantity, $\delta E<0$ for overcritical values of the magnetic field $B \geqslant B_{c 1}^{\mathrm{EW}}$. The condensation energy is shown in Fig. 3(right) for various values of the Higgs mass. Notice that a heavier Higgs boson makes a smaller contribution both to the $W$ condensate and to the energy density.

In Fig. 4 we visualize the absolute value of the $W$ condensate which is given by Eq. (64) with parameters (37). The $W$ condensate has a structure of the equilateral triangular (hexagonal) lattice in the transverse plane.

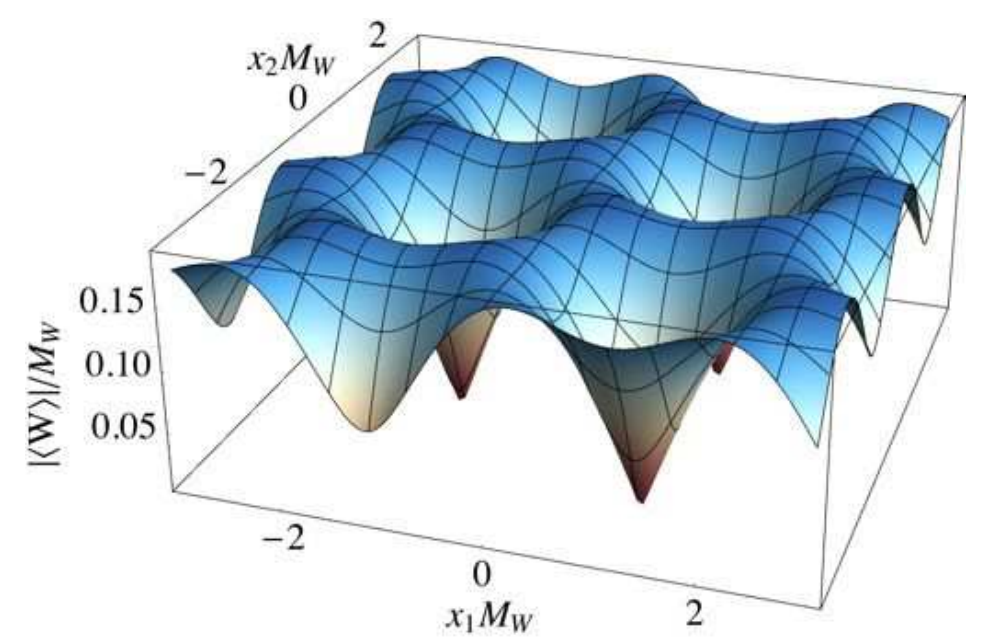

Fig. 4. The superconducting $W$ condensate (64) as a function of the transverse plane coordinates $x_{1}$ and $x_{2}$ at the physical Higgs mass ${ }^{71,72} M_{H}=125 \mathrm{GeV}$ in the background magnetic field $B=1.01 B_{c 1}^{\mathrm{EW}}$ directed along the $x_{3}$ axis. 


\subsubsection{Neutral and Higgs condensates}

Equations (63) and (56) imply that the magnetic field $B=B(z) \equiv B\left(x_{1}, x_{2}\right)$ is related to the $W$ condensate as follows:

$$
\partial\left(B-e|W|^{2} / 2\right)=0
$$

This relation is valid up to $O\left(\epsilon^{2}\right)$ terms where the parameter of smallness $\epsilon$ is given in Eq. (62). The solution of Eq. (66) is similar to the solution (38) in the GL model:

$$
B(z)=B_{\text {ext }}+\frac{e}{2}|W(z)|^{2}-\frac{e}{2}\left\langle|W|^{2}\right\rangle_{\perp},
$$

where the integration constant, given by the last term, guarantees the conservation of the total magnetic flux passing through the transverse plane,

$$
\int_{\mathcal{A}} d z d \bar{z} B(z)=\operatorname{Area}(\mathcal{A}) \cdot B_{\text {ext }}
$$

Thus, the magnetic field (67) becomes a transversally nonuniform function due to the backreaction of the inhomogeneous $W$ condensate (64).

It is interesting to note that the magnetic-field-induced $W$ condensation diminishes the value of the Higgs condensate and, simultaneously, induces an inhomogeneous condensation of the $Z$ bosons. Using the solution (67) for the magnetic field $B \equiv F_{12}$, one can solve Eqs. (58) and (57) and obtain the following nonlocal expressions for the $Z$ and Higgs condensates, respectively:

$$
\begin{aligned}
Z \equiv Z_{1}+i Z_{2} & =\frac{g \cos \theta}{2 i} \frac{\partial}{-\Delta+M_{Z}^{2}}|W|^{2}, \\
\phi & =\frac{v}{\sqrt{2}}\left(1-\frac{g^{2}}{4} \frac{1}{-\Delta+M_{H}^{2}}|W|^{2}\right),
\end{aligned}
$$

where $\Delta \equiv \bar{\partial} \partial=\partial_{1}^{2}+\partial_{2}^{2}$ is the two-dimensional Laplacian in the transverse plane. The remaining equation of motion (59) is satisfied automatically up to $O\left(\epsilon^{2}\right)$ terms.
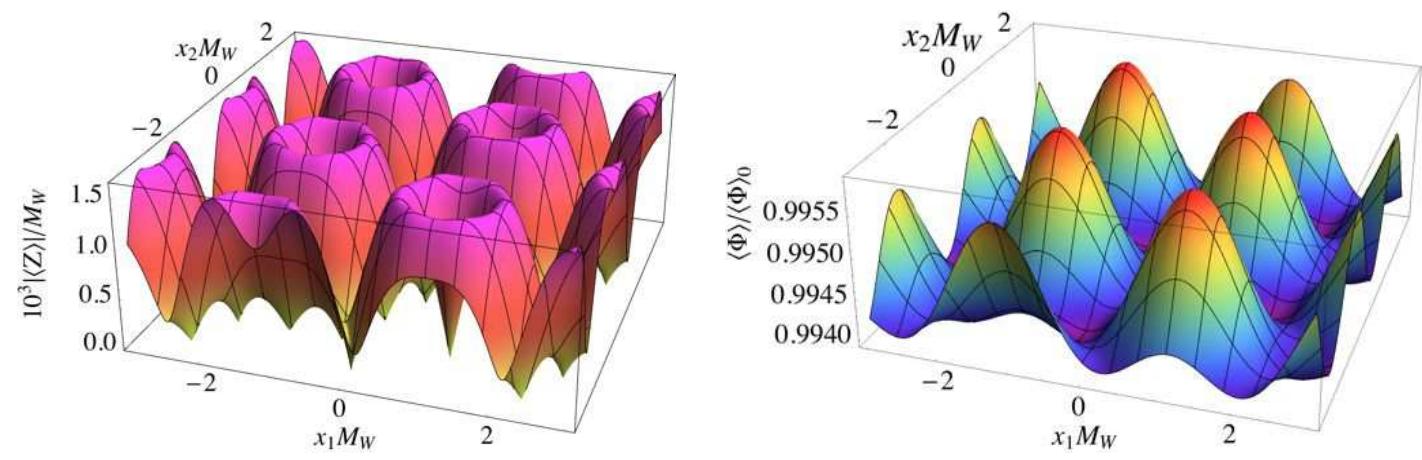

Fig. 5. The same as in Fig. 4 but for (left) the superfluid $Z$ condensate (69); (right) the expectation value of the Higgs field (70) divided by the $B_{\text {ext }}=0$ expectation value $\langle\Phi\rangle_{0}$. 
In the ground state at $B>B_{c 1}^{\mathrm{EW}}$, the $Z$ condensate (69) and the Higgs condensate (70) are inhomogeneous periodic functions of the transversal coordinates $x_{1}$ and $x_{2}$ as shown in Fig 5 . The $Z$ condensate has a quite complicated structure in the transverse plane. The Higgs condensate gets suppressed due to the presence of the $W$ condensate. A comparison of Fig. 4 with Fig. 5(right) shows that the the Higgs condensate takes larger values in the vicinity of the points where the $W$ condensate vanishes.

The overall effect of the magnetic field on the Higgs condensate can clearly be seen from Fig. 6. In the vicinity of the phase transition the Higgs condensate is a linearly diminishing function of the magnetic field. The slope of this function becomes weaker as the Higgs mass increases. Notice that near the phase transition the effect of the magnetic field on the expectation value of the Higgs field is very small: at the magnetic field exceeding the critical value $B_{c}^{\mathrm{EW}}$ by $10 \%$ the Higgs condensate gets decreased only by $1 \%$. However, we will see below that the dependence of the Higgs condensate on magnetic field becomes more pronounced as the magnetic field increases. Eventually, at certain critical magnetic field the Higgs condensate vanishes and a new symmetric phase forms.

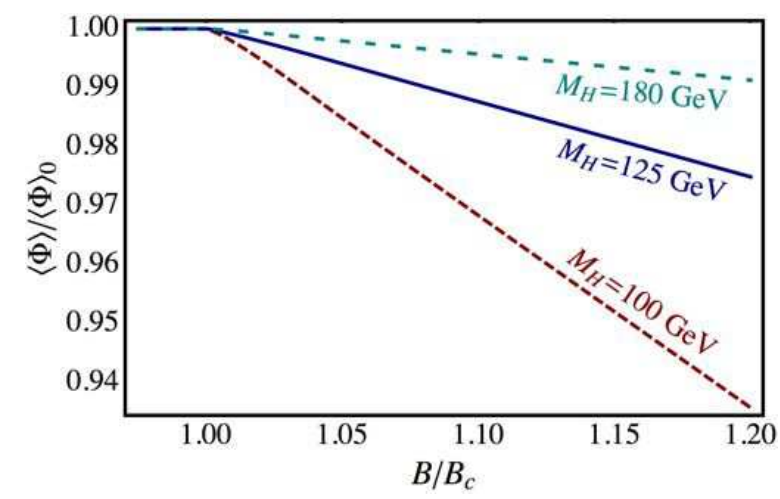

Fig. 6. The cell-averaged Higgs expectation value $\langle\Phi\rangle \equiv\left\langle\Phi^{\dagger} \Phi\right\rangle^{1 / 2}$, Eq. (141), in units of the $B_{\text {ext }}=0$ expectation value $\langle\Phi\rangle_{0}$ vs. the strength of the magnetic field $B$ for various Higgs masses $M_{H}$. The Higgs condensate corresponding to the physical value of the Higgs mass is shown by the solid line.

\subsubsection{Kaleidoscopic vortex structure of the $W$ and $Z$ condensates}

The topological structure of the $W$ and $Z$ condensates is quite nontrivial. As one can already notice from Figs. 4 and 5 (left) both charged $W=W_{1}^{-}+i W_{2}^{-}$and neutral $Z=Z_{1}+i Z_{2}$ condensates vanish at a set of points which form a regular lattice structure in the transverse plane. Moreover, as we will see below the phases of these fields acquire the contribution $\pm 2 \pi$ as one circumvents these points. Therefore, the behavior of the condensates around these points is similar to the one of the Cooper-pair field $\phi(x)$ around the Abrikosov vortices in the GL model (14). Thus, 
we associate the singularities in the charged $W$ and neutral $Z$ fields with superconducting and superfluid vortices, respectively. These superconductor and superfluid vortices should be distinguished from the existing $W$ - and $Z$ - electroweak vortex solutions, ${ }^{73}$ including known solutions which carry electric currents along vortex cores. $^{74,75}$

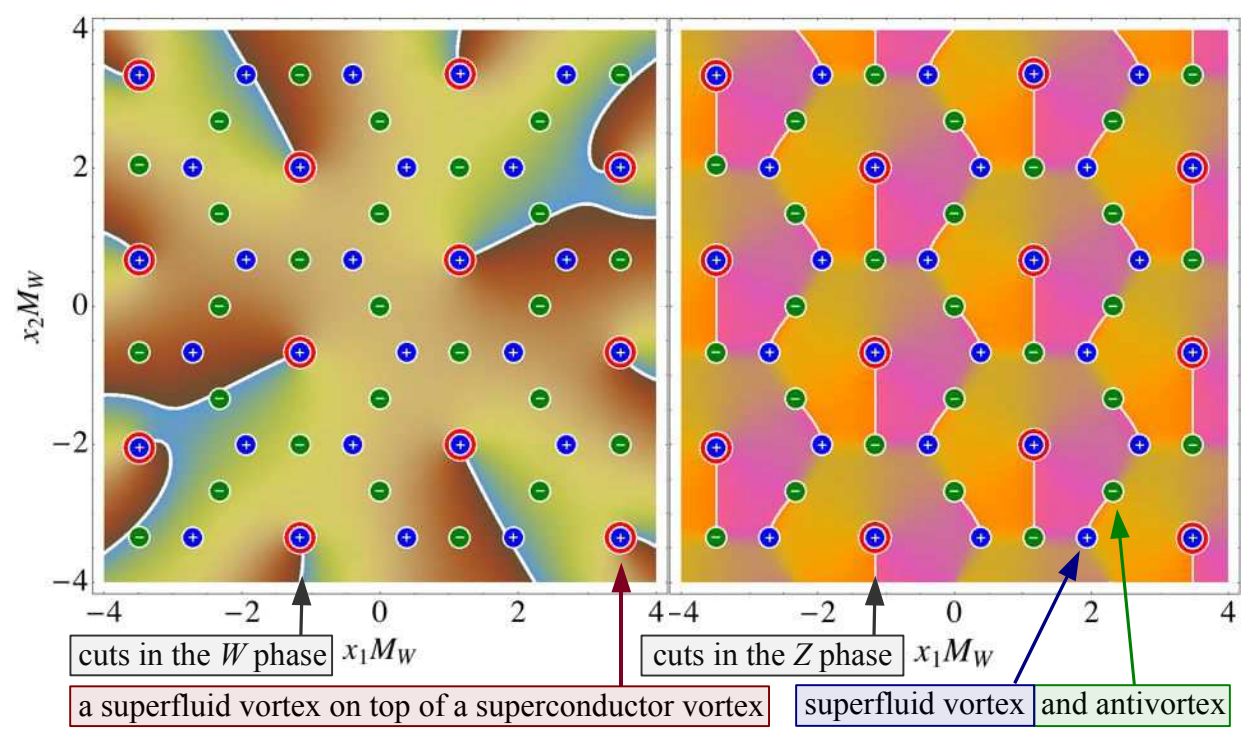

Fig. 7. The density plot of the phase of (the left panel) the $W$ condensate (64); and (right panel) the $Z$ condensate $(69)$ in the transversal $\left(x_{1}, x_{2}\right)$ plane at $B_{\text {ext }}=1.01 B_{c}^{\mathrm{EW}}$. The white lines correspond to the $2 \pi$ discontinuities in the phases of these condensates. The end points (shown by the circles) of the discontinuity lines are the superconductor vortices and superfluid (anti)vortices, respectively (from Ref. [54]).

In three dimensional coordinate space all vortices are parallel to the magnetic field axis. In the transverse plane they form a complicated kaleidoscopic pattern which is visualized in Fig. 7 along with the density plots of the phases of both condensates. The phases of the corresponding condensates changes by $2 \pi$ at the white lines in Fig. 7 (from the point of view of three spatial dimensions the $2 \pi$ singularities are two-dimensional Dirac sheets). The positions of these (Dirac) lines are gauge-dependent and hence unphysical. However, the positions of the end-points of the Dirac lines are physical as they correspond to the gauge-invariant topological vortices and antivortices.

It is clearly seen from Fig. 7(left) that the superconducting vortices - denoted by the large red circles - form an equilateral triangular (hexagonal) lattice. ${ }^{46-48,53}$ The defects in the $W$ condensate corresponds to vortices while the superconducting antivortices are absent. Each vortex is an endpoint of a Dirac line which starts at a vortex position and ends at spatial infinity.

The ground state has a much more complicated structure in the neutral sector. The density plot of the phase of the neutral $Z$ condensate, Fig. 7 (right), shows that the Dirac lines are finite segments with two endpoints, each of which correspond 
either to a superfluid vortex or to an antivortex. The vortex-antivortex pairs form a hexagonal structure superimposed onto the hexagonal lattice of the superconducting vortices. Notice that certain superfluid vortices are located at the superconductor vortices while the superfluid antivortices appear always alone.

\subsubsection{Nondissipative transport in the ground state}

The presence of the charged and neutral condensates suggest that the electroweak vacuum at $B>B_{c 1}^{\mathrm{EW}}$ may possess superconducting and superfluid features, respectively. In order to check this suggestion it is sufficient to calculate a response of the ground state with respect to an infinitesimally weak perturbation given by a test electric field $E^{\text {ext }}$. To this end let us consider electrically charged current $J_{\mu}^{E}$ and electrically neutral current $J_{\mu}^{Z}$ :

$$
\begin{aligned}
& J_{\mu}^{E}=\partial^{\nu} F_{\nu \mu} \equiv \frac{\delta \mathcal{L}}{\delta A^{\mu}}, \\
& J_{\mu}^{Z}=\partial^{\nu} Z_{\nu \mu} \equiv \frac{\delta \mathcal{L}}{\delta Z^{\mu}} .
\end{aligned}
$$

Using the classical equations of motion (53) one can show that in the presence of the static uniform electric field $E^{\text {ext }}$ these currents obey the following relations,

$$
\begin{array}{ll}
\partial_{[0} J_{3]}^{E}(x)=-\kappa^{E}\left(x_{1}, x_{2}\right) \cdot E_{3}^{\mathrm{ext}}, & \partial_{[0} J_{i]}^{E}=0, \\
\partial_{[0} J_{3]}^{Z}(x)=-\kappa^{Z}\left(x_{1}, x_{2}\right) \cdot E_{3}^{\mathrm{ext}}, & \partial_{[0} J_{i]}^{Z}=0,
\end{array}
$$

where $i=1,2$. The right hand sides of Eqs. (73) and (74) are described by the functions

$$
\begin{aligned}
& \kappa^{E}\left(x_{1}, x_{2}\right)=e^{2}|W|^{2}\left(x_{1}, x_{2}\right), \\
& \kappa^{Z}\left(x_{1}, x_{2}\right)=-e^{2} \cot \theta \cdot \frac{\Delta}{-\Delta+M_{Z}^{2}}|W|^{2}\left(x_{1}, x_{2}\right) .
\end{aligned}
$$

which characterize the transport properties of the electrically charged $\kappa^{E}$ and electrically neutral $\kappa^{Z}$ currents, respectively.

Strikingly, Eq. (73) implies the anisotropic superconductivity of the ground state at $B>B_{c 1}^{\mathrm{EW}}$ : a weak electric field introduces a resistance-free growth of electric current which continues streaming after the electric field is switched off. The effect is anisotropic because the superconductivity is realized only in the direction of the external magnetic field $B$. In addition, Eq. (74) implies an anisotropic superfluidity of the neutral $Z$ currents, and it illustrates a very unusual physical effect: an external electric field induces a current $J^{Z}$ of neutral particles which are flowing frictionlessly along the magnetic field axis. One can also show that a weak transverse electric field $E_{1,2}^{\text {ext }}$ induces neither superconducting nor superfluid currents. ${ }^{54}$

It is interesting to notice that from the point of view of the electric conductivity properties, a ground state of the vacuum can be either a superconductor or an insulator due to Lorentz symmetry. A dissipative behavior described by the Ohm's 
Law is excluded because is inconsistent with the Lorentz symmetry of the vacuum. Indeed, the formulation of the Ohm's Law, $\boldsymbol{J}=\sigma \boldsymbol{E}$, requires a presence of a definite "time" direction since the left-hand side of the Ohm's equation is a vector while the right-hand-side is a component of a field strength tensor. Thus, the absence of the electric resistance in the superconducting $B>B_{c 1}^{\mathrm{EW}}$ phase is protected by a remnant Lorentz symmetry in the $\left(x_{0}, x_{3}\right)$ plane. Similar Lorentz-protection arguments are applied to the superfluid property as well.

The transport coefficients (75) and (76) are shown in Fig. 8 as functions of the transverse coordinates $x_{1}$ and $x_{2}$. A comparison of Fig. 8(left) with Fig. 4 shows that the superconductivity is suppressed in the cores of the superconducting vortices.
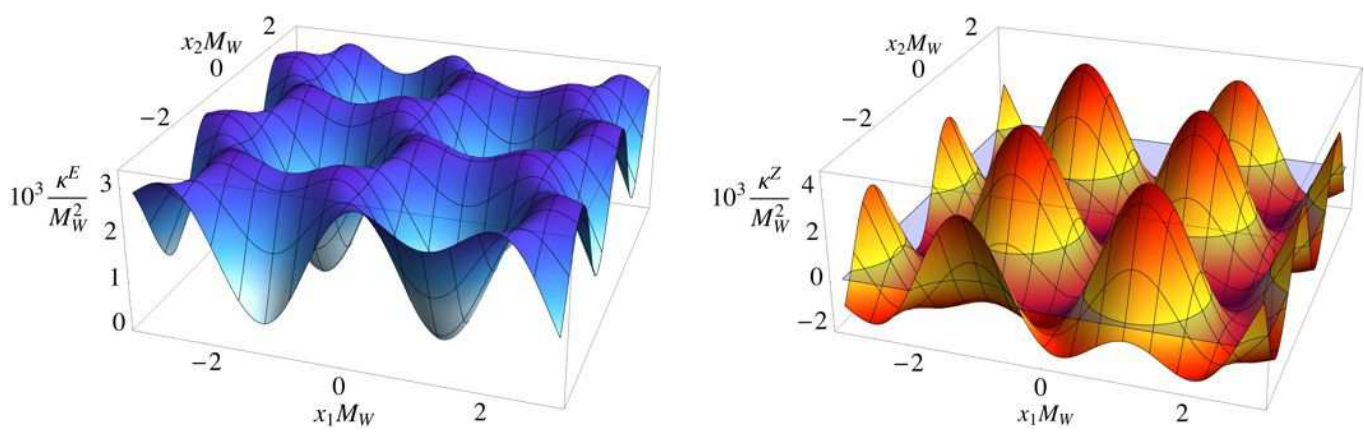

Fig. 8. (Left) The superconducting (75) and (right) superfluid (76) transport coefficients as the functions of the transverse plane coordinates $x_{1}$ and $x_{2}$ at the physical Higgs mass $M_{H}=125 \mathrm{GeV}$ in the background magnetic field $B=1.01 B_{c}^{\mathrm{EW}}$ directed along the $x_{3}$ axis.

In the vicinity of the phase transition at $B>B_{c 1}^{\mathrm{EW}}$ the superconductivity coefficient $(75)$, averaged over the transversal $\left(x_{1}, x_{2}\right)$ plane,

$$
\bar{\kappa}^{E} \equiv\left\langle\kappa^{E}\right\rangle_{\perp}=\frac{1}{\operatorname{Area}(\mathcal{A})} \int_{\mathcal{A}} d x_{1} d x_{2} \kappa^{E}\left(x_{1}, x_{2}\right)
$$

is a linearly growing function of the magnetic field $B$, Fig. 9. The superfluid coefficient (76) is a sign-changing function, Fig. 8(right), of the transversal coordinates $x_{1,2}$ which has a vanishing mean value after the averaging over the transversal plane, $\bar{\kappa}^{Z} \equiv\left\langle\kappa^{E}\right\rangle_{\perp}=0$. The sign-changing nature of the superfluidity coefficient $\kappa^{Z}$ in Eq. (74) implies that the electric current induces the superfluid flows both along and opposite to the direction of the magnetic field. The sign of the superfluid current depends on the coordinates $\left(x_{1}, x_{2}\right)$ in the transverse plane.

The spatial distribution of the electric and neutral currents flowing along the magnetic field axis can be read off from the corresponding superconducting coefficients in Fig. 8. An illustration of the induced currents in shown in Fig. 10.

Thus, we conclude that a weak external electric field $E_{3}^{\text {ext }}$ applied along the magnetic field in the condensed phase gives rise to ballistically growing

(i) net electric current along the magnetic field axis, and

(ii) inhomogeneous neutral flow with vanishing net current. 


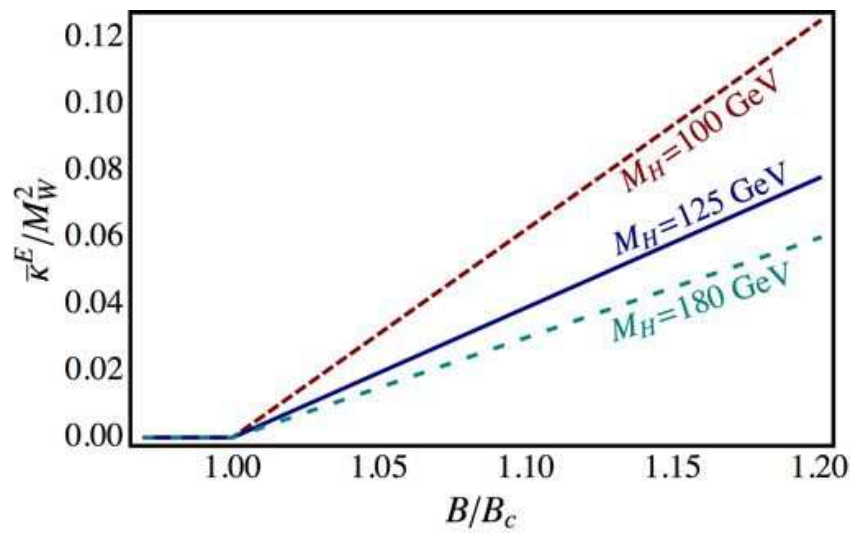

Fig. 9. The cell-averaged superconductivity transport coefficient (75), given in Fig. 8(left), vs. the magnetic field $B$ at various fixed values of the Higgs masses. The cell-averaged superfluidity coefficient, Fig. 8(right), is always zero.

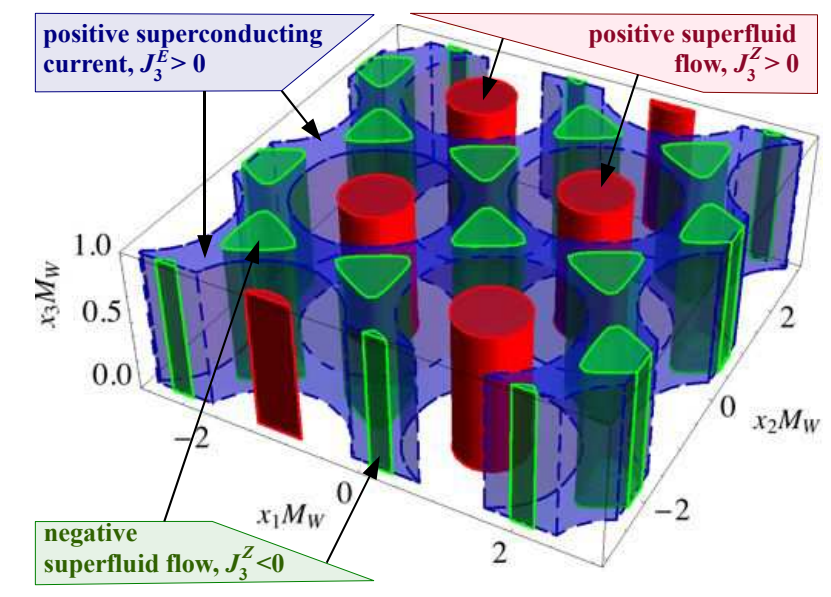

Fig. 10. The three-dimensional regions of space predominantly occupied by the superconducting electric current $J_{3}^{E}$ of the $W$ bosons and the superfluid neutral flows $J_{3}^{Z}$ of the $Z$ bosons generated by a weak test electric field $E_{\text {ext }}>0$ parallel to the strong magnetic field $B=1.01 B_{c}^{\mathrm{EW}}$ (from Ref. [54]).

Thus, the ground state of electroweak vacuum at $B>B_{c 1}^{\mathrm{EW}}$ is a "tandem" phase which is, simultaneously, an electromagnetic superconductor and a neutral superfluid. The superfluid currents are dragged by the superconducting currents, so that the superfluidity is a secondary effect. As we discuss below, the same phenomenon may also exist in QCD.

\subsection{Symmetry restoration at the second critical magnetic field}

\subsubsection{Structure of the symmetry restored phase}

We have seen in Fig. 6 that the Higgs condensate $\langle\Phi\rangle$ is a diminishing function of the magnetic field strength in the vicinity of the first critical field (50). As the magnetic field increases, the Higgs condensate diminishes further and eventually 
vanishes as the magnetic field reaches its second critical value (61). At this point the electroweak symmetry gets completely restored. ${ }^{55,69,70}$

The restoration of the electroweak symmetry at high magnetic field can be deduced from the classical equations of motion. First, we have found that above the first critical field (50) the $W$ condensate is spontaneously formed. Second, according to Eq. (57), the $W$ condensate suppresses the equilibrium (minimum) value of the Higgs potential towards smaller values of the Higgs field. When the second critical value (61) is approached, the Higgs expectation value becomes small, and it eventually vanishes.

Notice, however, that the directions of the $W$ and Higgs condensates are locked with each other as the direction in the group space of the latter defines the one for the former. In other words, the $W$ field is an offdiagonal $S U(2)_{L}$ vector field with respect to the Higgs field condensate $\langle\Phi\rangle$ which identifies the diagonal direction according to the symmetry breaking pattern (44). Thus, we are coming to the conclusion that exactly at the second critical magnetic field $B=B_{c 2}^{\mathrm{EW}}$, the Higgs field vanishes and the direction of the $W$ field in the color space becomes undefined. Strictly speaking, at the second critical magnetic field a restoration of the color symmetry takes place and the expectation value of the $W$ field must vanish as well, $\langle W\rangle\left(B>B_{c 2}^{\mathrm{EW}}\right) \equiv 0$ because the $W$ condensate may point towards any direction in the group space. Thus we are coming to the eventual paradox that in order to achieve the symmetry restored phase with $\langle\Phi\rangle=0$ one needs a strong $W$ condensate while the symmetries of this new phase forbid the presence of the $W$ condensate at all.

The mentioned paradox may be resolved if one takes into account the fact that the expectation value of the Higgs field is affected not only by the $W$ condensate but also by a flux of the $Z$ field. ${ }^{46,47}$ In the vicinity of the $B \gtrsim B_{c 1}^{\mathrm{EW}}$ the $Z$-flux is small and well defined relative to the large Higgs condensate - and thus it was neglected in our considerations above - while in the vicinity of the second phase transition the Z-flux becomes ill-defined and one should decompose this Z-flux into its nonAbelian $S U(2)$ and hypercharge $U(1)$ constituents. Both quantities are of the same order as the $W$ field that will be cancelled by the former at the phase transition. The external Abelian magnetic field induces thus magnetic fluxes associated with the $\mathrm{U}(1)$ hypercharge bosons $X_{\mu}$ which, in turn, suppress the vacuum expectation value of the Higgs field in the symmetric phase.

At the quantum level the paradox can be resolved in a more elegant way with the help of nonperturbative quantum effects. First, we notice that in the symmetry restored vacuum the dynamics of the non-Abelian $W$ fields becomes similar to the dynamics of the gluon fields in the vacuum of Quantum Chromodynamics where the color symmetry is unbroken. Second, we mention that although the color symmetry of the QCD vacuum is never broken, the gluon fields are nevertheless condensed due to certain quantum effects. The best description of this effect can be found in the scope of the "spaghetti vacuum" picture of the QCD vacuum which is also known 
as the Copenhagen vacuum.

In the spaghetti vacuum picture the vacuum of QCD is considered to be populated by evolving vortex tubes which carry a chromomagnetic flux. ${ }^{56-58,76,77}$ The standard mechanism of formation of the chromomagnetic vortices is as follows. The perturbative vacuum of QCD - which is paramagnetic due to the asymptotic freedom - has an unstable mode towards formation of a chromomagnetic field. ${ }^{78}$ However, in the background of a homogeneous chromomagnetic field the gluon part of the vacuum energy develops an imaginary part due to large chromomagnetic moment of the gluon ${ }^{76}$ in a similar to the discussed mechanism of the generation of the $W$ condensate in the superconducting phase of the electroweak theory. This property implies in turn that the homogeneous chromomagnetic field is also unstable towards forming an inhomogeneous state of separate parallel flux tubes carrying the chromomagnetic field (chromomagnetic vortices), ${ }^{58}$ similarly to the Abrikosov vortex lattice in a mixed state of an ordinary type-II superconductor in an external magnetic field. ${ }^{62}$ Finally, due to the global rotational and Lorentz invariance of the QCD vacuum, the chromomagnetic field must have a local domain-like structure: ${ }^{77}$ the gluonic field has different color and spacial orientations in different domains. Since the vortices follow the direction of the chromomagnetic field, the vortex lines form an intertwining entangled structure, hence the name "spaghetti".

Coming back to the electroweak theory, the mentioned analogy with the QCD vacuum suggests that in the magnetic-field-induced symmetric phase of the electroweak vacuum the $W$ condensate is a large quantity in local domains of the space-time. Due to the large local value of the $W$ condensate the Higgs condensate is always zero so that the electroweak symmetry is restored everywhere despite the global expectation value of the $W$ field is zero. The electroweak vacuum in the symmetry-restored phase at $B>B_{c 2}^{\mathrm{EW}}$ should be populated by the tube-like structures made of the $W$ fields which possess arbitrary orientation in the $S U(2)_{L}$ group space. As the magnetic field is lowered slightly below the second critical field, $B<B_{c 2}^{\mathrm{EW}}$, the symmetry breaking (44) takes place leading to a definite reorientation of the $W$ fields into the off-diagonal - with respect to the Higgs expectation value - subgroup of the non-Abelian gauge group. This approach to the electroweak structure unifies the QCD Copenhagen picture, and the structure of the electroweak vacuum both in the the superconducting phase and in the symmetryrestored phase. ${ }^{55}$

\subsubsection{Vortex solutions}

The vortex solutions in the vicinity of the second critical field were obtained in Ref. [55]. In this region the value of the Higgs field is a small quantity, $|\langle\Phi\rangle| \ll M_{H}$, and in the leading order the classical equations of motion are becoming equivalent to the classical vacuum equations for a pure $S U(2)_{L}$ theory,

$$
\overline{\mathfrak{D}} W=0, \quad g w_{12}^{3}=\frac{g^{2}}{2}|W|^{2},
$$


where

$$
w_{\mu \nu}^{3}=\partial_{\mu} W_{\nu}^{3}-\partial_{\nu} W_{\mu}^{3},
$$

is the Abelian field strength for the diagonal component of the $W$ field. Although the small Higgs field does not enter explicitly the equations of motion, the presence of this field reflected by the choice of the Abelian subgroup in the $S U(2)_{L}$ gauge group: ${ }^{55}$

$$
\left\langle w_{12}^{3}\right\rangle_{\perp}=e B_{\text {ext }} .
$$

The solutions of Eqs. (78) and (79) were first addressed in. ${ }^{57,58} \mathrm{~A}$ more accurate approach was given in, ${ }^{59}$ where solutions for rectangular lattices in terms of Weierstrass elliptic functions are obtained. The case of a hexagonal vortex lattice which is expected to be a true ground state - was considered in Ref. [55].

Equations (78) can be rewritten in a complexified form as follows

$$
\begin{aligned}
\bar{\partial} \ln W & =-i g \bar{W}^{3}, \\
\partial \ln W^{*} & =i g W^{3}, \\
i g\left(\bar{\partial} W^{3}-\partial \bar{W}^{3}\right) & =-g^{2}|W|^{2} .
\end{aligned}
$$

These three equations can further be reduced down to the following two equations

$$
\begin{aligned}
\Delta \ln |W|^{2}+g^{2}|W|^{2} & =0, \\
\Delta \arg (W)+\frac{g}{2}\left(\bar{\partial} W^{3}+\partial \bar{W}^{3}\right) & =0 .
\end{aligned}
$$

The phase of the $W$ condensate, $\arg (W)$, becomes a harmonic function in the gauge

$$
\partial \cdot W^{3}=\bar{\partial} W^{3}+\partial \bar{W}^{3}=0,
$$

because in this case the second term of Eq. (85) vanishes. The exact form of this function - up to local gauge transformations - is fixed by topology of underlying vortex lattice.

The solution of the Liouville equation (84) corresponding to a hexagonal lattice of chromomagnetic vortices with unit winding was found in Ref. [55]:

$$
g W(z, \bar{z})=\frac{2 \sqrt{2}|w|}{\left|\vec{y}^{\dagger}(\bar{z}) \cdot M \cdot \vec{y}(z)\right|} \frac{\sigma^{*}(\bar{z})}{|\sigma(z)|},
$$

where $y_{i}(z)=Y_{i}\left(4 \mathcal{P}^{3}(z) \lambda^{6}\right), \mathcal{P}$ and $\sigma$ the Weierstrass $\mathcal{P}$ and $\sigma$ functions. The constant $|w| \approx 1 / 4$ and the matrix $M$ is

$$
M=\frac{\left(\begin{array}{cc}
Y_{2}(1) Y_{2}(0) & -Y_{1}(1) Y_{2}(0) \\
-Y_{2}(0) Y_{1}(1) & Y_{1}(0) Y_{1}(1)
\end{array}\right)}{\sqrt{Y_{2}(1) Y_{2}(0) Y_{1}(1) Y_{1}(0)-\left[Y_{2}(0) Y_{1}(1)\right]^{2}}}
$$

where the functions

$$
\begin{aligned}
& Y_{1}(X)={ }_{2} \mathrm{~F}_{1}\left(\frac{1}{4},-\frac{1}{12} ; \frac{2}{3} ; X\right), \\
& Y_{2}(X)=\lambda \cdot{ }_{2} \mathrm{~F}_{1}\left(\frac{1}{4},-\frac{1}{12} ; \frac{1}{2} ; 1-X\right),
\end{aligned}
$$


are determined via the Gaussian hypergeometric function ${ }_{2} \mathrm{~F}_{1}$. The constant $\lambda$ determines the physical scale of the solution,

$$
\lambda=\frac{2 \pi L_{B}}{\nu \Gamma^{3}(1 / 3)},
$$

where $L_{B}$ and $\nu$ are given in Eqs. (25) and (37), respectively. Despite of the very complicated form of the solution (87)-(91) around the second critical magnetic field, its visual representation - up to a suitable redefinition of the physical scales - is almost undistinguishable from the perturbative solution around the first critical field shown in Fig. 4.

Thus, the electroweak model at zero temperature exhibits an unusual phase structure at nonzero magnetic field characterized by the presence of insulating, superconducting and symmetry-restored phases (Fig. 2). The strength of the relevant magnetic fields is of the order of the electroweak scale $e B_{\text {ext }} \sim 10^{20} \mathrm{~T}$. In the next section we discuss a possible realization of the superconducting phase in a weaker magnetic fields of the order of the QCD scale $10^{16} \mathrm{~T}$.

\section{Superconducting phase in QCD}

Recently it was suggested that a sufficiently strong magnetic field of hadronic scale may cause the vacuum to behave as an inhomogeneous and anisotropic electromagnetic superconductor. ${ }^{35,38}$ The superconductivity of, basically, empty space, should be caused by a spontaneous creation of charged $\rho$-meson condensates if the strength of the magnetic field exceeds the critical value

$$
B_{c}^{\mathrm{QCD}} \simeq 10^{16} \mathrm{~T} \quad \text { or } \quad e B_{c}^{\mathrm{QCD}} \simeq 0.6 \mathrm{GeV}^{2} .
$$

The vacuum superconductivity at the QCD scale is similar to its magnetic-fieldinduced counterpart in the electroweak theory which is caused by the condensation of the $W$ bosons. The effect is accompanied by a superfluid-like condensation of the neutral $\rho$ mesons similarly to the inhomogeneous condensation of the $Z$ bosons.

The charged $\rho$ mesons - or, better to say, quark-antiquark condensates with the quantum numbers of the $\rho$ mesons - play a central role in the suggested superconducting mechanism in QCD. The $\rho$ mesons are vector particles with anomalously high magnetic moment corresponding to the gyromagnetic ratio $g=2$. One can argue that the anomalous magnetic moment provides a large negative contribution to the squared energy of the $\rho$ mesons in a background of strong magnetic fields. As the external magnetic field exceeds the critical value of the magnetic field (92), the energy becomes purely imaginary indicating a condensation of the $\rho$ mesons. The emergence of the electrically charged condensate implies, almost inevitably, electromagnetic superconductivity of the new vacuum ground state. ${ }^{35}$

One can also argue that in the background of the strong magnetic field the charged vector mesons play the role of the Cooper pairs: the strong magnetic field makes the motion of the quarks essentially one dimensional because the electrically charged quarks may move only along the lines of the magnetic field. In one spatial 
dimension a weak attraction between a quark (for example, "up" quark) and an antiquark (say, "down" antiquark) mediated by a (virtual) gluon inevitably leads to creation of a bound state, electrically charged vector meson (in our example, it is $\rho^{+} \equiv u \bar{d}$ meson). The emergence of the bound states leads to lowering of the vacuum energy and, again, to condensation of the charged $\rho$ mesons.

The superconductivity of the vacuum in a strong magnetic field was first found in an effective bosonic model which describes the electrodynamics of the $\rho$ mesons. ${ }^{35}$ Later, the superconductivity effect was confirmed in the Nambu-JonaLasinio model ${ }^{79}$ in Ref. $[38,39]$. Signatures of this unusual effect were also found in holographic approaches ${ }^{40-43}$ and in numerical simulations of quenched lattice QCD. ${ }^{44}$ A possibility of the $\rho$-meson condensation was also briefly mentioned in Ref. [80].

Due to the anisotropic nature of the superconductivity (the vacuum superconducts only along the axis of the magnetic field) the Meissner effect is absent so that the $\rho$-meson condensate does not screen the external magnetic field. ${ }^{35}$ Moreover, due to the anisotropic superconductivity the vacuum may behave analogously to a (hyperbolic) metamaterial which, electromagnetically, shares similarity with diffractionless "perfect lenses". ${ }^{37}$

Below we review our current understanding of the ground state of zerotemperature QCD in strong magnetic field.

\subsection{Effective mean-field approach to QCD in strong magnetic field}

\subsubsection{Electrodynamics of $\rho$ mesons}

The $\rho$ meson is a vector (spin-1) particle made of a light (up or down) quark and a light (down or up) antiquark. Since this problem is of a nonperturbative nature, its first-principle treatment starting from the QCD Lagrangian is so far absent. Therefore in this section we discuss the vacuum superconductivity within a scope of an effective model given by a self-consistent quantum electrodynamics for the charged and neutral $\rho$ mesons. The model is described by Djukanovic-SchindlerGegelia-Scherer (DSGS) Lagrangian: ${ }^{81}$

$$
\begin{aligned}
\mathcal{L}= & -\frac{1}{4} F_{\mu \nu} F^{\mu \nu}-\frac{1}{2} \rho_{\mu \nu}^{\dagger} \rho^{\mu \nu}+m_{\rho}^{2} \rho_{\mu}^{\dagger} \rho^{\mu} \\
& -\frac{1}{4} \rho_{\mu \nu}^{(0)} \rho^{(0) \mu \nu}+\frac{m_{\rho}^{2}}{2} \rho_{\mu}^{(0)} \rho^{(0) \mu}+\frac{e}{2 g_{s}} F^{\mu \nu} \rho_{\mu \nu}^{(0)}
\end{aligned}
$$

which extends the vector meson dominance model ${ }^{82}$ with the Maxwellian $U(1)$ sector by adding all allowed interactions of both charged, $\rho_{\mu} \equiv \rho^{-}=\left(\rho_{\mu}^{(1)}-i \rho_{\mu}^{(2)}\right) / \sqrt{2}$ and $\rho_{\mu}^{+}=\rho_{\mu}^{\dagger}$, and neutral, $\rho_{\mu}^{(0)}$, mesons with the electromagnetic field $A_{\mu}$. The 
tensor quantities in (93) describe various strength tensors,

$$
\begin{aligned}
F_{\mu \nu} & =\partial_{\mu} A_{\nu}-\partial_{\nu} A_{\mu}, \\
f_{\mu \nu}^{(0)} & =\partial_{\mu} \rho_{\nu}^{(0)}-\partial_{\nu} \rho_{\mu}^{(0)}, \\
\rho_{\mu \nu}^{(0)} & =f_{\mu \nu}^{(0)}-i g_{s}\left(\rho_{\mu}^{\dagger} \rho_{\nu}-\rho_{\mu} \rho_{\nu}^{\dagger}\right), \\
\rho_{\mu \nu} & =D_{\mu} \rho_{\nu}-D_{\nu} \rho_{\mu},
\end{aligned}
$$

where the covariant derivative is

$$
D_{\mu}=\partial_{\mu}+i g_{s} \rho_{\mu}^{(0)}-i e A_{\mu},
$$

and $g_{s} \equiv g_{\rho \pi \pi} \approx 5.88$ is the $\rho \pi \pi$ vertex coupling.

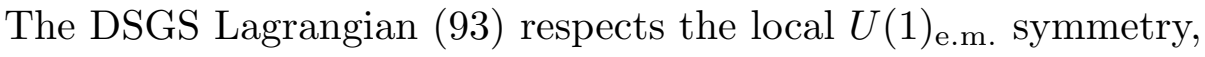

$$
U(1)_{\mathrm{e} . \mathrm{m} .}: \quad\left\{\begin{array}{l}
\rho_{\mu}(x) \rightarrow e^{i \omega(x)} \rho_{\mu}(x), \\
A_{\mu}(x) \rightarrow A_{\mu}(x)+\partial_{\mu} \omega(x),
\end{array}\right.
$$

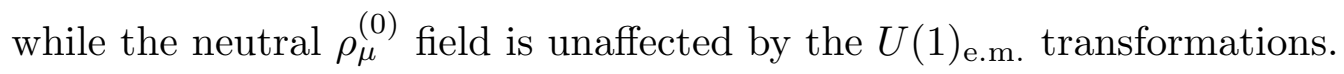

\subsubsection{The $\rho$-meson condensation due to strong magnetic field}

The last term of the DSGS Lagrangian (93) describes a nonminimal coupling of the $\rho$ mesons to the electromagnetic field which implies the anomalously large gyromagnetic ratio $(g=2)$ of the charged $\rho^{ \pm}$mesons.

In a simple way the condensation can be explained as follows. ${ }^{35}$ In the background of a strong enough magnetic field a spin-one particle with gyromagnetic ratio $g=2$ should experience a tachyonic instability towards formation of a BoseEinstein condensate. Namely, a free charged relativistic spin-1 particle with the gyromagnetic ratio $g=2$ and the mass $m$ has the following energy spectrum in a background of an external magnetic field $\vec{B}_{\text {ext }}=\left(0,0, B_{\text {ext }}\right)$ :

$$
\varepsilon_{n, s_{z}}^{2}\left(p_{z}\right)=p_{z}^{2}+\left(2 n-2 s_{z}+1\right) e B_{\text {ext }}+m^{2} .
$$

where $s_{z}=-1,0,+1$ is the spin projection on the field's axis $\hat{z} \equiv \hat{x}_{3}, n \geqslant 0$ is a nonnegative integer number (which, together with $s_{z}$, labels the Landau levels), and $p_{z}$ is the particle momentum along the field's axis. The ground state (with $n=0$, $s_{z}=+1, p_{z}=0$ ) has the following (squared) energy:

$$
\varepsilon_{0}^{2}\left(B_{\text {ext }}\right)=m^{2}-e B_{\text {ext }}
$$

which can be associated with the ground state mass of a vector particle in strong magnetic field. The lowest energy of the charged $\rho$-meson in the external magnetic field becomes purely imaginary quantity if the magnetic field exceeds the critical value $B_{c}=m^{2} / e$.

Exactly the same result, Eq. (101), can be derived from the DSGS model (93) in a strong magnetic field background. ${ }^{35}$ The condensed state corresponds to the following $\rho$-meson wavefunction:

$$
\rho_{1}\left(x_{\perp}\right)=-i \rho_{2}\left(x_{\perp}\right)=\rho\left(x_{\perp}\right), \quad \rho_{0}=\rho_{3}=0 .
$$


At the level of quark wavefunction the new ground state is described by the following vector quark-antiquark condensates:

$$
\left\langle\bar{u} \gamma_{1} d\right\rangle=\rho\left(x_{\perp}\right), \quad\left\langle\bar{u} \gamma_{2} d\right\rangle=i \rho\left(x_{\perp}\right) .
$$

The quark-antiquark condensates (103) carry the quantum numbers of the electrically charged $\rho$ mesons so that this phenomenon may also be interpreted as the $\rho$-meson condensation.

In the absence of an external magnetic field the $\rho$ meson is an unstable particle. All known decays of the $\rho^{ \pm}$mesons are going via the modes: ${ }^{83}$

$$
\rho^{ \pm} \rightarrow \pi^{ \pm} X, \quad X=\pi^{0}, \eta, \gamma, \pi \pi \pi .
$$

The fraction of the primary decay mode, $X=\pi^{0}$, is greater than $99 \%$.

A consequence of the diminishing mass of the $\rho$ meson in strong magnetic field is a stabilization of the $\rho$ meson due to kinematical reasons. Indeed, as the strength of the background magnetic field increases, the product of the decay, the charged pion [which is always produced in the known decay modes of the $\rho^{ \pm}$mesons (104)] becomes heavier ${ }^{27}$ while the decaying particle, the lowest state of the $\rho^{ \pm}$meson, becomes lighter (101). Obviously, at a certain magnetic field $B_{\rho^{ \pm}} \simeq 0.36 B_{c}^{\mathrm{QCD}}$ the masses of the initial and final states in the dominant channel, $\rho^{ \pm} \rightarrow \pi^{ \pm} \pi^{0}$, should become equal, and the fast strong decays (104) of the charged $\rho$ mesons should eventually become impossible. ${ }^{35}$ Similar arguments can be applied to the neutral $\rho$ mesons.

At $B_{\text {ext }}>B_{c}^{\mathrm{QCD}}$ the system experiences a tachyonic instability towards the Bose-Einstein condensation of the spin-1 particles. A similar condensation effect of the $W$-bosons was discussed in the previous section in the context of the electroweak model in a background with a much stronger magnetic field. ${ }^{46-48}$ Another example is represented by a pure Yang-Mills theory, in which the gluons may condense in a chromomagnetic field background. ${ }^{56}$

The condensation of the $\rho$ mesons in the vacuum should take place at the critical strength of the magnetic field (92) defined by the $\rho$-meson mass $m_{\rho}=775.5 \mathrm{MeV}$. Similarly to the condensation of the $W$ bosons in the electroweak model, the condensation of the $\rho$ mesons should lead to an electromagnetic superconductivity of the QCD vacuum. Below we discuss the details of this condensation.

\subsubsection{Equations of motion of the effective model}

A variation of the DSGS Lagrangian (93) with respect to the electromagnetic potential $A_{\mu}$ provides us with the Maxwell-type equation of motion,

$$
\partial^{\nu} F_{\nu \mu}=-J_{\mu}
$$

where the electric current $J_{\mu}$ consists of two conserved currents

$$
J_{\mu}=J_{\mu}^{\mathrm{ch}}+J_{\mu}^{(0)}
$$


coming from the charged and neutral mesons,

$$
\begin{aligned}
J_{\mu}^{\mathrm{ch}} & =i e\left[\left(D_{\mu} \rho^{\nu}\right)^{\dagger} \rho_{\nu}-\rho^{\nu \dagger} D_{\mu} \rho_{\nu}+\partial^{\nu}\left(\rho_{\nu}^{\dagger} \rho_{\mu}-\rho_{\mu}^{\dagger} \rho_{\nu}\right)+\rho_{\nu}^{\dagger} D^{\nu} \rho_{\mu}-\left(D^{\nu} \rho_{\mu}\right)^{\dagger} \rho_{\nu}\right],(10 \\
J_{\mu}^{(0)} & =-\frac{e}{g_{s}} \partial^{\nu} f_{\nu \mu}^{(0)}
\end{aligned}
$$

respectively.

A variation of the DSGS Lagrangian (93) with respect to the field $\rho_{\mu}^{(0)}$ gives us the second equation of motion,

$$
\left(\partial^{\nu} \partial_{\nu}+m_{\rho^{(0)}}^{2}\right) \rho_{\mu}^{(0)}-\partial_{\mu} \partial^{\nu} \rho_{\nu}^{(0)}-\frac{g_{s}}{e} J_{\mu}^{\mathrm{ch}}=0
$$

along with the following conservation law: $\partial^{\mu} \rho_{\mu}^{(0)}=0$.

Equation (109) encodes with the mass of the neutral $\rho^{(0)}$ meson:

$$
m_{0} \equiv m_{\rho^{(0)}}=m_{\rho}\left(1-\frac{e^{2}}{g_{s}^{2}}\right)^{-\frac{1}{2}} .
$$

Using Eqs. (106), (108) and (109) one can get a well-known relation between the electromagnetic current $J_{\mu}$ and the neutral meson field $\rho_{\mu}^{(0)}$ :

$$
J_{\mu}=\frac{e m_{0}^{2}}{g_{s}} \rho_{\mu}^{(0)},
$$

which emerged originally in the scope of vector dominance models long time ago. ${ }^{84}$

The third equation of motion is

$$
\left[\left(D^{\alpha} D_{\alpha}+m_{\rho}^{2}\right) g_{\mu \nu}-D_{\mu} D_{\nu}+i\left(g_{s} \rho_{\mu \nu}^{(0)}+g_{s} f_{\mu \nu}^{(0)}-2 e F_{\mu \nu}\right)\right] \rho^{\nu}=0,
$$

The linear part of Eq. (112) gives us the mass of the charged $\rho^{ \pm}$meson, $m_{\rho^{ \pm}}=m_{\rho}$. The neutral vector $\rho^{(0)}$ meson is heavier compared to its charged counterpart $\rho^{ \pm}$.

\subsubsection{Induced condensates and energy density of the ground state}

Let us consider the spontaneous condensation of the $\rho$ mesons in the background of the magnetic field (18) in the vicinity of the suggested phase transition: $B>B_{c}^{\mathrm{QCD}}$ with $B-B_{c}^{\mathrm{QCD}} \ll B_{c}^{\mathrm{QCD}}$. Since the external field is taken to be slightly stronger than the critical value (92), the $\rho$-meson condensate is small, $|\rho| \ll m_{\rho}$, so that the equations of motion for $\rho$-meson electrodynamics (93) can be linearized in analogy with our considerations in the electroweak model. Following the example of the Ginzburg-Landau model, we consider static $x_{3}$-independent solutions which may, however, be inhomogeneous in the transversal $\left(x_{1}, x_{2}\right)$ plane.

The equation for the $\rho$-meson condensate (102) in the overcritical magnetic field $\left(B \gtrsim B_{c}^{\mathrm{QCD}}\right)$ is similar to the one for the Cooper pair condensate in the subcritical magnetic field $\left(B \lesssim B_{C}^{\mathrm{QCD}}\right)$ in the Ginzburg-Landau model: ${ }^{61}$

$$
\mathfrak{D} \rho \equiv\left(\partial-\frac{e}{2} B_{\text {ext }} z\right) \rho=0,
$$

where the covariant derivative is given in Eq. (21) and the complex wavefunction $\rho=\rho(z)$ is defined in Eq. (102) 
Following our experience in the GL model we represent the solution of Eq. (113) for the $\rho$-meson condensate in a manner similar to Eqs. (23) and (64)

$$
\rho(z)=\sum_{n \in \mathbb{Z}} C_{n} h_{n}\left(\nu, \frac{z}{L_{B}}, \frac{\bar{z}}{L_{B}}\right)
$$

where the function $h_{n}$ is given in Eq. (24) and $L_{B}$ is the magnetic length (25). As usual, the coefficients $C_{n}$ are assumed to obey the $N$-fold symmetry (26).

The inhomogeneities in the $\rho$-meson condensate induce condensation of the neutral mesons, $\rho^{(0)}=\rho_{1}^{(0)}+i \rho_{2}^{(0)}$ :

$$
\rho^{(0)}\left(x_{\perp}\right)=\frac{2 i g_{s}}{-\partial_{\perp}^{2}+m_{0}^{2}} \partial|\rho|^{2}
$$

with $\rho_{0}^{(0)}=\rho_{3}^{(0)}=0$. In Eq. (115) $\partial_{\perp}^{2} \equiv \partial_{1}^{2}+\partial_{2}^{2}$,

$$
\frac{1}{-\partial_{\perp}^{2}+m_{0}^{2}}\left(x_{\perp}\right)=\frac{1}{2 \pi} K_{0}\left(m\left|x_{\perp}\right|\right),
$$

is the two-dimensional Euclidean propagator of a scalar particle with the mass of the neutral $\rho^{(0)}$ meson (110), and $K_{0}$ is a modified Bessel function. The condensation of the neutral $\rho^{(0)}$ mesons $(115)$ is analogous to the condensation of the inhomogeneous condensation of the $Z$ mesons in the electroweak model considered earlier.

The magnetic field is also an inhomogeneous function of the transversal coordinates,

$$
B\left(x_{\perp}\right)=B_{\text {ext }}+\frac{2 e m_{0}^{2}}{-\partial_{\perp}^{2}+m_{0}^{2}}\left[|\rho(z)|^{2}-\left\langle|\rho|^{2}\right\rangle_{\perp}\right],
$$

where the last term, defined in Eq. (28), guarantees the conservation of the magnetic flux (39). Equation (117) is a $\rho$-meson analogue of the GL relation (38).

Notice that in the vacuum subjected to the strong magnetic field, the neutral condensate (115) and the magnetic field (117) depend on the (charged) $\rho$-meson condensate nonlocally contrary to the local relation between the magnetic field and the Cooper pair condensate (38) in the GL model for the ordinary superconductivity.

The energy density of the vacuum in the presence of the $\rho$-meson condensate is given by the following formula:

$$
\begin{aligned}
\langle\mathcal{E}\rangle \equiv\left\langle T_{00}\right\rangle= & \frac{1}{2} B_{\text {ext }}^{2}+2\left(m_{\rho}^{2}-e B_{\text {ext }}\right)\left\langle|\rho|^{2}\right\rangle_{\perp}+2 e^{2}\left\langle|\rho|^{2}\right\rangle_{\perp}^{2} \\
& +2\left(g_{s}^{2}-e^{2}\right)\left\langle|\rho|^{2} \frac{m_{0}^{2}}{-\Delta+m_{0}^{2}}|\rho|^{2}\right\rangle_{\perp},
\end{aligned}
$$

where $T_{\mu \nu}$ is the energy-momentum tensor,

$$
T_{\mu \nu}=2 \frac{\partial \mathcal{L}}{\partial g^{\mu \nu}}-\mathcal{L} g_{\mu \nu}
$$

corresponding to the DSGS model (93), and the brackets $\langle\ldots\rangle_{\perp}$ indicate the average over the transversal $\left(x_{1}, x_{2}\right)$ plane (28). Notice that contrary to the GL model (27), the energy density of the $\rho$-meson condensate (118) contains a nonlocal positive $\left(g_{s} \gg e\right)$ quartic term. 


\subsubsection{Superconductor vortices: the lattice structure}

The quadratic term $\left\langle|\rho|^{2}\right\rangle$ in the energy density (118) can be evaluated with the help of Eq. (31), while - contrary to the energy density of the GL model (27) - the local quartic term $\left\langle|\rho|^{4}\right\rangle$ is absent in Eq. (118). Instead, the condensate $\rho$ is stabilized by a nonlocal quartic term, which is proportional to the following nonlocal functional:

$$
Q[\rho]=\left\langle|\rho|^{2} \frac{m_{0}^{2}}{-\Delta+m_{0}^{2}}|\rho|^{2}\right\rangle,
$$

which can conveniently be represented as follows:

$$
Q[\rho]=\frac{1}{\text { Area }_{\perp}} \int \frac{d^{2} k}{(2 \pi)^{2}} \frac{m^{2}}{k^{2}+m_{0}^{2}}|q(k ; \rho)|^{2},
$$

where

$$
q(k ; \rho)=\int d x_{1} \int d x_{2} e^{i k_{1} x_{1}+i k_{2} x_{2}}\left|\rho\left(x_{1}, x_{2}\right)\right|^{2},
$$

with $q^{*}(k ; \rho) \equiv q(-k ; \rho)$ and $\rho\left(x_{1}, x_{2}\right) \equiv \rho\left(x_{1}+i x_{2}\right)$.

The structure of the energy functional (118) indicates that an analogue of the Abrikosov ratio (36) in the case of the $\rho$-meson condensation is as follows:

$$
\beta_{\rho}=\left\langle\frac{|\rho|^{2}}{\left\langle|\rho|^{2}\right\rangle} \frac{m_{0}^{2}}{-\Delta+m_{0}^{2}} \frac{|\rho|^{2}}{\left\langle|\rho|^{2}\right\rangle}\right\rangle \equiv \frac{Q[\rho]}{\left\langle|\rho|^{2}\right\rangle^{2}} .
$$

The minimum of the energy functional (118) corresponds to the minimum of the new dimensionless parameter $\beta_{\rho}$. From Eqs. (120), (121) and (122) it is clear that the value of the new Abrikosov parameter is entirely encoded into the constants describing the $\rho$-meson condensate (114). Contrary to the Abrikosov ratio (36), the quantity (123) depends on the strength of the magnetic field $B$. In the "local" (and, unphysical) limit $m_{0} \rightarrow \infty$ the quantity (123) is reduced to the Abrikosov ratio (36).

The minimization of the mean energy density (118) as a function of (generally, complex) lattice parameters $C_{n}, n=1, \ldots, N$ was performed in Ref. [36] for a fixed set of values $N=1, \ldots, 8$. It turns out that the condensation energy reaches its minimum at the equilateral triangular lattice with $N=2$ and $C_{0}=i C_{1}$, similarly to the case of the Abrikosov lattice in the GL model. All lattices with odd values of $N$ possess higher energies while all even $-N$ lattices are reduced to the $N=2$ case.

In Fig. 11 we show the parameter $\beta_{\rho}$, Eq. (123), as a function of the magnetic field $B$ in the vicinity of the critical magnetic field $B_{c}^{\mathrm{QCD}}$. The minimum of the quantity $\beta_{\rho}$ and, as a consequence, the minimum of the energy density functional (118), are reached at $N=2$ for all studied values of the magnetic field. Due to the specific values of the phenomenological parameters of the DSGS model (93) - which describes the electrodynamics of the $\rho$-meson excitations in the QCD vacuum - the difference in energies between visually different lattices is tiny. For example, at $B=1.01 B_{c}^{\mathrm{QCD}}$ the difference in the condensation energies between the square, $N=1$, lattice and the equilateral triangular, $N=2$, lattice is less than $0.5 \%$. The relative difference in the corresponding dimensionless $\beta$ parameters is of the same 


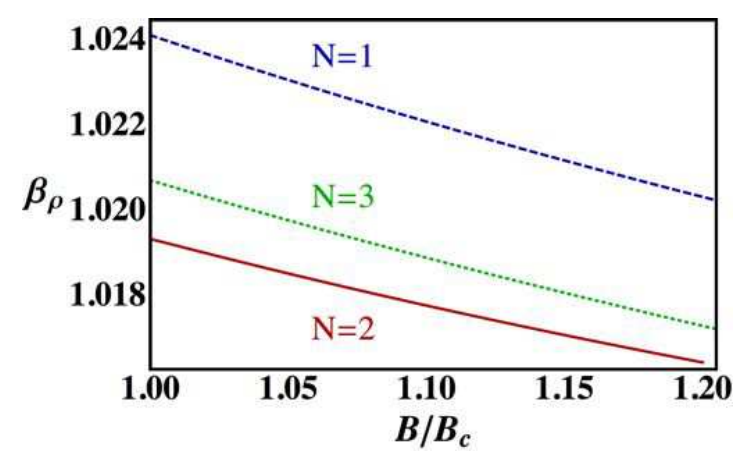

Fig. 11. The parameter $\beta_{\rho}$, Eq. (123), for different types of lattices, $N=1,2$, 3, as a function of the magnetic field $B$. The $N=2$ solution corresponds to the absolute minimum of the mean energy density.

order. Since the difference in most important bulk parameters (e.g., average energy, mean conductivity etc) between the square lattice and its possible conformations is very small, the square lattice anyway is a very good approximation for calculation of the bulk properties of the real vacuum state.

\subsubsection{Charged vector condensate and energy density}
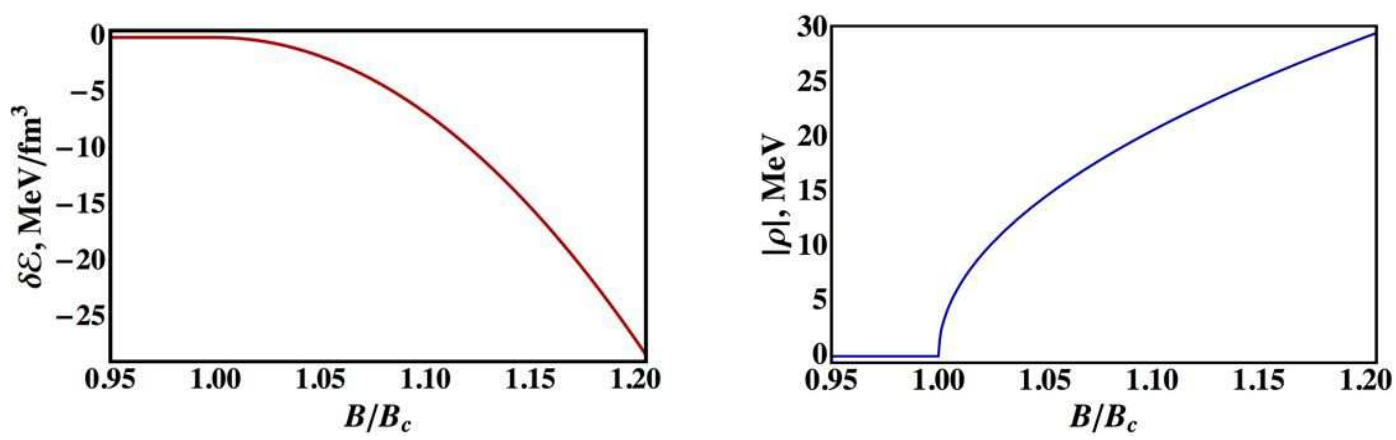

Fig. 12. At $B>B_{c}^{\mathrm{QCD}}$ the superconducting state is more energetically favorable compared to the trivial vacuum state: (left) the condensation energy becomes negative due to emergence of the superconducting condensate (124), $|\rho| \equiv \sqrt{\left\langle|\rho|^{2}\right\rangle}$ (right) at the critical magnetic field $B=$ $B_{c}^{\mathrm{QCD}}$ with $B_{c}^{\mathrm{QCD}}$ given in Eq. (92). The quantities are shown for the minimal-energy (equilateral triangular) lattice structure.

The mean condensation energy density is shown in Fig. 12 (left) as a function of the magnetic field. One can clearly see that at $B<B_{c}^{\mathrm{QCD}}$ the condensation energy is zero while it becomes negative at $B>B_{c}^{\mathrm{QCD}}$ due to condensation of the charged $\rho$ mesons. In order to characterize the later property, we notice that for the equilateral triangular vortex lattice the mean squared superconducting condensate is related to the coefficient $C_{0}$ of the solution (114) as follows:

$$
\left\langle|\rho|^{2}\right\rangle \equiv \frac{1}{\text { Area }_{\perp}} \int d^{2} x|\rho(x)|^{2}=\frac{1}{2 \sqrt[4]{3}}\left|C_{0}\right|^{2} .
$$


The mean of the squared condensate (124) is shown in Fig. 12 (right) as a function of the magnetic field. It is clear that at $B>B_{c}^{\mathrm{QCD}}$ the superconducting state with a nontrivial condensate $\rho \neq 0$ is energetically more favorable compared to the trivial vacuum state with $\rho=0$. The stronger magnetic field the larger the gain in energy due to the condensation of the $\rho$ mesons.
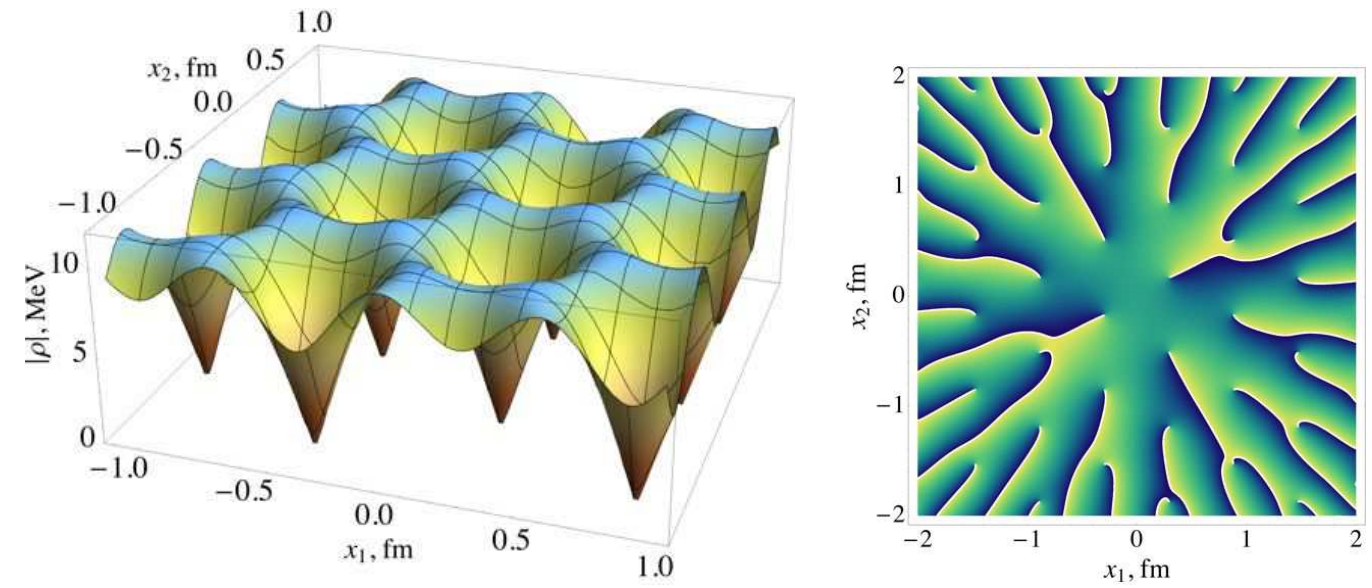

Fig. 13. (Left) The absolute value of the superconducting condensate $\rho$, Eq. (114) and (right) the density plot of its phase $\varphi_{\rho}=\arg \rho$ at $B=1.01 B_{c}^{\mathrm{QCD}}$ in the transversal $\left(x_{1}, x_{2}\right)$ plane. In the density plot the white lines corresponds to the cuts in the phase. The endpoints of the cuts mark the positions of the superconductor $\rho$ vortices.

The vortex structure of the superconducting ground state can easily be seen from the behavior of the superconducting order parameter $\rho$. Similarly to the vortex pattern in the GL model, the $\rho$ condensate has a characteristic form $\rho(z, \bar{z}) \propto z-z_{0}$ near the position $z_{0}=x_{1,0}+i x_{2,0}$ of each elementary vortex. At the vortex core $z=z_{0}$ the condensate should vanish linearly. Moreover, in the local vicinity of the point $z_{0}$ the phase $\varphi_{\rho}=\arg \rho$ of the condensate should behave as a polar angle. Thus, the phase $\varphi_{\rho}$ winds around the position of the vortex and the winding corresponds to a topological stability of the vortex. Since the phase is defined modulo $2 \pi$, the phase $\varphi_{\rho}$ should experience cuts in the $\left(x_{1}, x_{2}\right)$ plane. At branches of these cuts the phase $\varphi_{\rho}$ experiences the quantized jumps, $\varphi_{\rho} \rightarrow \varphi_{\rho} \pm 2 \pi$.

The absolute value and the phase of the superconducting condensate are shown in Fig. 13. The phase of the condensate experiences $2 \pi$ jumps at the one-dimensional semi-infinite curves in the transverse plane which start at the points where the condensate $\rho$ is vanishing, and end at spatial infinity. The position and the shape of the cuts can be changed by the $U(1)$ gauge transformations (99), while the endpoints of the cuts are gauge invariant quantities corresponding to the superconductor vortices $^{\mathrm{a}}$ which organize themselves into the equilateral triangle lattice.

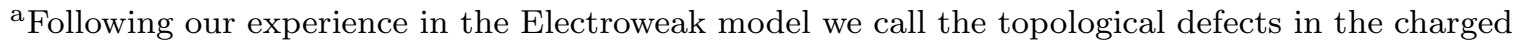
$\rho$ condensate as "superconductor/superconducting vortices" in order to distinguish them from
} 


\subsubsection{Electric currents and superconductivity}

The electric current density can be derived from Eq. (93):

$$
J_{\mu}=i e\left[\rho^{\nu \dagger} \rho_{\nu \mu}-\rho^{\nu} \rho_{\nu \mu}^{\dagger}+\partial^{\nu}\left(\rho_{\nu}^{\dagger} \rho_{\mu}-\rho_{\mu}^{\dagger} \rho_{\nu}\right)\right]-\frac{e}{g_{s}} \partial^{\nu} f_{\nu \mu}^{(0)} .
$$

In the ground state the longitudinal components of the electric current are vanishing, $J_{0}=J_{3}=0$, while the transversal current,

$$
J_{\perp}\left(x_{\perp}\right) \equiv J_{1}\left(x_{\perp}\right)+i J_{2}\left(x_{\perp}\right)
$$

becomes a nonlocal function of the superconducting condensate: ${ }^{35}$

$$
J_{\perp}\left(x_{\perp}\right)=2 i e m_{0}^{2} \cdot\left(\frac{\partial}{-\partial_{\perp}^{2}+m_{0}^{2}}|\rho|^{2}\right)\left(x_{\perp}\right) .
$$

The nonlocal nature of the relation between the transversal electric current (127) and the charged condensate $\rho$ is an important distinction between the QCD vacuum in the superconducting phase and the GL superconductor in the mixed phase (1).

The electric current (127) is a persistent current of the charged $\rho$-meson degrees of freedom. The current originates from the quarks and antiquarks which popup from the virtual state and form a condensate with the quantum numbers of the charged $\rho$-meson. This current is always present in the superconducting phase at $B>B_{c}^{\mathrm{QCD}}$ and it vanishes in the normal phase of the vacuum.
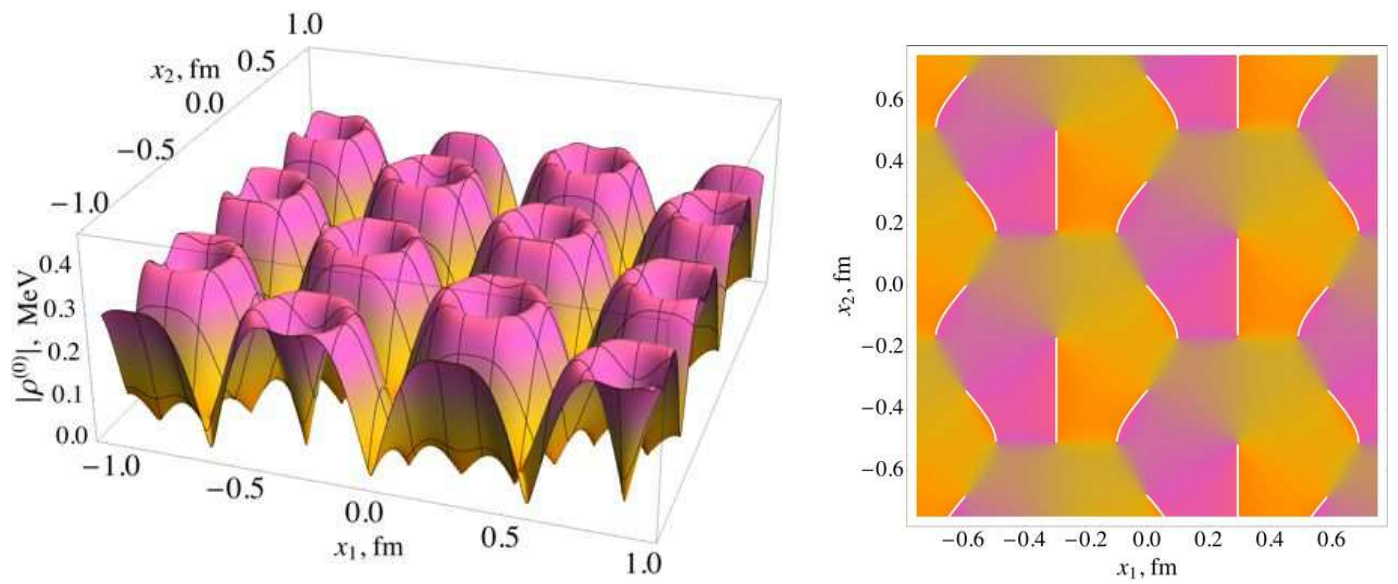

Fig. 14. (left) Absolute value of the superfluid condensate $\rho^{(0)}$, Eq. (115), and (right) the density plot of its phase at $B=1.01 B_{c}^{\mathrm{QCD}}$ in the transversal $\left(x_{1}, x_{2}\right)$ plane.

In Fig. 14(left) we show the absolute value of the neutral condensates $\rho^{(0)}$ as a function of the transverse coordinates $x_{1}$ and $x_{2}$ for a slightly overcritical background magnetic field, $B=1.01 B_{c}^{\mathrm{QCD}}$. One can compare this figure with Fig. 13 where the charged condensate is plotted for the same set of parameters. Firstly, we

"superfluid vortices" which are similar vortexlike defects in the neutral $\rho^{(0)}$ condensate. 
notice that the neutral condensate is much weaker compared to its charged counterpart, $\left|\rho^{(0)}\right| \ll|\rho|$. Secondly, the geometrical pattern of the neutral condensate is much more involved compared to the charged one. In particular one observes that the neutral $\rho$-meson condensate vanishes in a denser, triangular set of points compared to its superconducting counterpart. As the phases of the neutral $\rho^{(0)}$ field are winding around these points, they correspond to superfluid vortices and antivortices, depending on the direction of winding.

In Fig. 14(right) we show the density plot of the phase of the neutral $\rho$-meson field (115) in the transverse plane $\left(x_{1}, x_{2}\right)$. The white lines correspond to the cuts in the phase, so that the phase of the neutral condensate winds around the endpoints of these lines. At these endpoints the absolute value of the neutral field vanishes and the phase becomes undefined. Thus, the endpoints correspond to the (superfluid) vortices in the neutral $\rho$-meson field. Similarly to the structure of the ground state of electroweak model, the superfluid vortices and antivortices in QCD come always in pairs so that the net vorticity of the neutral field is always zero.
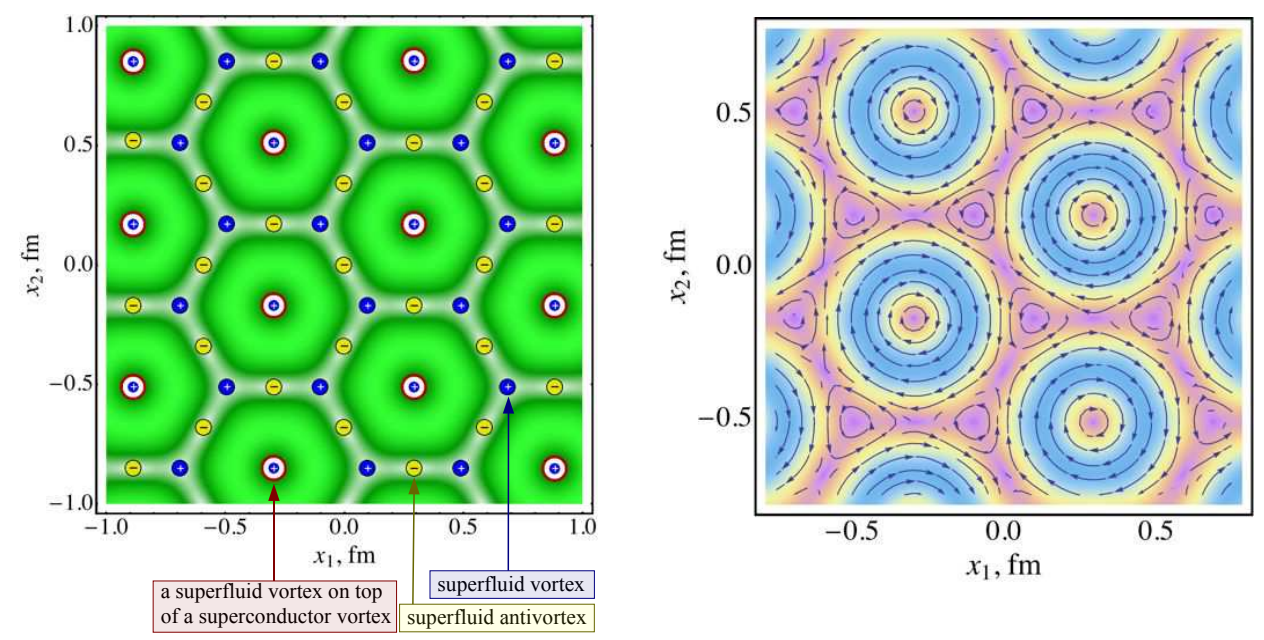

Fig. 15. (Right) The periodic vortex structure of the vacuum ground state is superimposed on the density plot (shown in shades of the green color) of the absolute value of the neutral $\rho$-meson condensate (115) at the magnetic field $B=1.01 B_{c}^{\mathrm{QCD}}$ (from Ref. [36]). Each superconductor vortex (the large red circles) is always superimposed on a superfluid vortex (the small blue disks marked by the plus signs) forming an equilateral triangular lattice. The isolated superfluid vortices and antivortices (the small yellow disks with the minus sign) arrange themselves in the hexagonal lattice pattern. (Left) The electric currents of the charged quark condensates in the ground state lattice at $B=1.01 B_{c}^{\mathrm{QCD}}$ in the transversal $\left(x_{1}, x_{2}\right)$ plane. The reddish (grayish) areas corresponds to the stronger (weaker) current.

The overall vortex structure of the suggested vacuum state is shown in Fig. 15(left). In this figure the vortex locations are superimposed on the density plot of the absolute value of the neutral meson field (115). The vortex pattern is quite remarkable as it shared many similarities with the kaleidoscopic picture of the electroweak vacuum in strong magnetic field. First of all, the superconductor vor- 
tices organize themselves in an equilateral triangular lattice. The superfluid vortices and antivortices are organized in a honeycomb (hexagonal tiling) pattern. A center of each hexagon is occupied by a superconductor vortex while each superconductor vortex is always superimposed on a superfluid vortex. In each lattice cell, one superconductor vortex is accompanied by three superfluid vortices and three superfluid antivortices so that the net superfluid vorticity per unit cell is always zero. It is interesting to notice that the vortex structure of the ground state, shown in Fig. 15(left), matches the pattern of the persistent transverse electric current (127) shown in Fig. 15(right).

\subsubsection{Anisotropic superconductivity of the ground state}

In transversal directions the strong magnetic field confines the local electric currents of charged condensates to the hexagonal cells, Fig. 15(right). The size of cells is of the order of the lowest Landau level of the $\rho$-meson condensate, that is of the order of the magnetic length $(25)\left(L_{B} \sim 0.6 \mathrm{fm}\right.$ at $\left.B \approx B_{c}^{\mathrm{QCD}}\right)$. A relatively week $(|\vec{E}| \ll|\vec{B}|)$ electric field $\vec{E} \perp \vec{B}$ cannot create a transversal ("intra-cell") electric current because such a current would involve an excitation at a first Landau level which is separated from a lowest Landau level by a large energy gap of the order of $\sqrt{|e B|}$. Thus, the global electric currents are suppressed in the transverse directions. This is a qualitative reason why the Meissner effect is absent in the superconducting ground state ${ }^{35}$ so that the emerging superconductivity does not screen the external magnetic field. A similar effect is predicted for the exotic magnetic-field-induced "reentrant" superconductivity in a type-II superconductor. ${ }^{66}$

The longitudinal electric currents may be nonvanishing because the motion of the quarks along the axis of the magnetic field is not restricted. If one applies a weak electric field $\vec{E}=\left(0,0, E_{3}\right)$ parallel to the strong magnetic field $\vec{B} \equiv(0,0, B)$ then the electric currents - induced by the external field - satisfy a modified London equation: ${ }^{35}$

$$
\frac{\partial J_{3}(x)}{\partial x_{0}}-\frac{\partial J_{0}(x)}{\partial x_{3}}=-\kappa\left(x_{\perp}\right) E_{3}
$$

while the induced transverse electric currents are obviously zero. The transverse current $J_{\perp}$ is unmodified by the external electric field directed along the magnetic field axis, Eqs. (126) and (127), and

$$
\frac{\partial J_{k}(x)}{\partial x_{\mu}}-\frac{\partial J_{\mu}(x)}{\partial x_{k}} \equiv 0,
$$

with $\mu=0, \ldots, 3$ and $k=1,2$.

The London-type equation is typical for superconducting systems as it characterizes a conducting state without resistance. The superconductivity is characterized by the quantity $\kappa=\kappa\left(x_{\perp}\right)$, which is a nonlocal function of the superconducting condensate:

$$
\kappa\left(x_{\perp}\right)=4 e^{2} m_{0}^{2} \cdot\left(\frac{1}{-\partial_{\perp}^{2}+m_{0}^{2}}|\rho|^{2}\right)\left(x_{\perp}\right) .
$$


In the superconducting ground state the "superconducting transport coefficient" $\kappa$ has a hexagonal lattice structure, Fig. 16. Due to nonlocal nature of the relation between the transport coefficient $\kappa$ and the superconducting condensate (130), the superconductivity is not completely suppressed inside the vortices. This property distinguishes the vacuum superconductivity in QCD from an ordinary one. The same property is shared by the superconductivity coefficient in the electroweak model as one can see from Eqs. (73) and (75) and Fig. 8(left). Contrary to QCD, in the GL model of superconductivity the mentioned relation between the superconducting coefficient and the density of the superconducting condensate is local and therefore the suppression of the condensation in the center of the Abrikosov vortex implies stronger suppression of the superconductivity in the vortex cores.

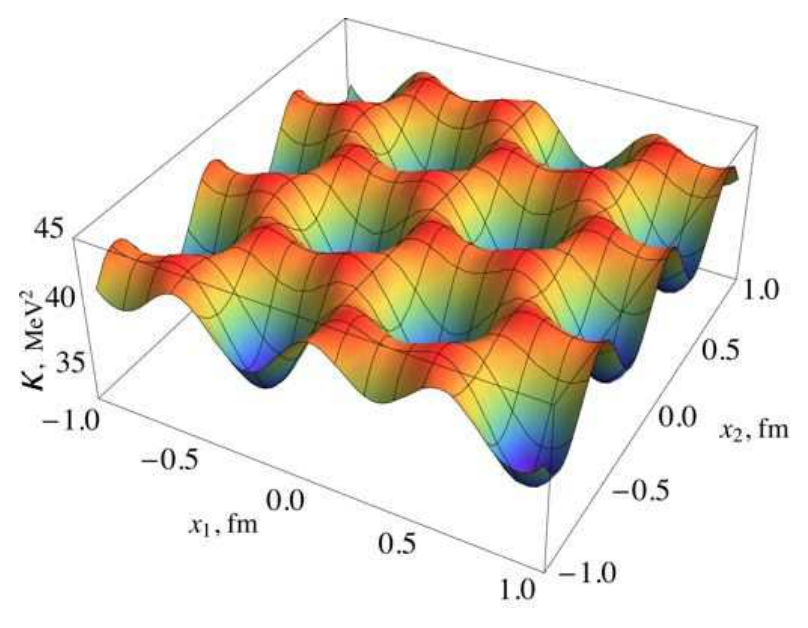

Fig. 16. The superconductivity coefficient $\kappa$, Eq. (130), in the modified London law (128), is shown as a function of $x_{1}$ and $x_{2}$ transverse coordinates at the magnetic field $B=1.01 B_{c}^{\mathrm{QCD}}$.

Thus, at high magnetic field the vacuum becomes an anisotropic inhomogeneous superconductor. The superconductivity is a strongly anisotropic feature of the system since the vacuum can superconduct along the direction of the magnetic field only while in the transversal directions the vacuum does not behave as superconductor. Moreover, the superconductivity is inhomogeneous due to the coordinate dependence of the corresponding transport coefficient $\kappa$, Eq. (130), Fig. 16.

Thus, according to the effective $\rho$-meson electrodynamics, the qualitative properties of the QCD vacuum are very similar to the features of the ground state of the electroweak model in the superconducting phase. One should mention that the condensation of the charged (superconducting) field $\rho_{\mu}$ leads to the induced condensation of a neutral, superfluid-like field $\rho_{\mu}^{(0)}$ in the ground state of the vacuum. ${ }^{35}$ The longitudinal components of the neutral condensate are zero, $\rho_{0}^{(0)}=\rho_{3}^{(0)}=0$, while the transverse components of the neutral condensate, are nonvanishing according to Eq. (115). These components should lead to the "tandem" superfluid properties of the $\rho^{(0)}$ condensate which are identical, at the qualitative level, to the properties 
of the superfluid $Z$ bosons. The only essential quantitative difference between the superconducting state in the electroweak model and in QCD appears to be in a $10^{4}$ fold difference in scales of the corresponding critical magnetic fields (50) and (61), and in $10^{2}$ fold difference in the magnetic lengths (25) which determine the size of the elementary vortex cells in the transverse plane.

\subsubsection{Symmetries of QCD ground state in the superconducting phase}

Let us first consider the symmetries of QCD in the suggested superconducting phase. The Lagrangian of the two-flavor QCD in the background of the electromagnetic field $A_{\mu}^{\mathrm{em}}$ reads as follows:

$$
\mathcal{L}=-\frac{1}{4} G_{\mu \nu}^{a} G^{a \mu \nu}-\bar{\psi}\left(i \gamma^{\mu} D_{\mu}-m\right) \psi,
$$

where $G_{\mu \nu}^{a}$ is the strength tensor of the gluon field $A_{\mu}^{a}$,

$$
D_{\mu}=\partial_{\mu}-i g T^{a} A_{\mu}^{a}-i q A_{\mu}^{\mathrm{em}}
$$

is the covariant derivative, $T^{a}$ are generators of the $S U(3)_{c}$ color group and

$$
q=\frac{e}{2}\left(\tau_{3}+\frac{1}{3}\right)
$$

is the electric charge matrix acting in the flavor space. For simplicity, the masses of up and down quarks are taken to be the same, $m_{u}=m_{d}=m$.

Due to the difference in electric charges of up and down quarks (133), $q_{u}=$ $2 e / 3$ and $q_{d}=-e / 3$, the group of the internal continuous global symmetries of Lagrangian (131) is explicitly broken by the background electromagnetic field $A_{\mu}^{\mathrm{em}}$ :

$$
S U_{V}(2) \times U_{B}(1) \rightarrow U(1)_{I_{3}} \times U(1)_{B}
$$

where $U(1)_{I_{3}}$ is the diagonal subgroup of the isospin group $S U(2)_{V}$ and $U(1)_{B}$ is the baryon number symmetry.

The internal local symmetries of Lagrangian (131) include the electromagnetic $U(1)$ em gauge symmetry

$$
U(1)_{\mathrm{em}}:\left\{\begin{array}{rl}
A_{\mu}^{\mathrm{em}}(x) & \rightarrow A_{\mu}^{\mathrm{em}}(x)+\partial_{\mu} \omega_{\mathrm{em}}(x) \\
\psi_{f}(x) & \rightarrow e^{i \omega_{\mathrm{em}}(x) q_{f}} \psi_{f}(x)
\end{array},\right.
$$

and the color $S U(3)_{c}$ gauge symmetry. These local symmetries are not anomalously broken so that the fermion determinant is invariant under the local $S U(3)_{c} \times U(1)_{\mathrm{em}}$ gauge group. The background magnetic field itself does not break explicitly the electromagnetic gauge symmetry (135) since the magnetic field is defined by a component of the gauge invariant Abelian field strength tensor $F_{\mu \nu}^{\mathrm{em}}=\partial_{\mu} A_{\nu}^{\mathrm{em}}-\partial_{\nu} A_{\mu}^{\mathrm{em}}$. In other words, QCD in a classical magnetic field background is invariant under the $U(1)_{\mathrm{em}}$ gauge transformations (135). Thus the Abelian symmetry (135) is the symmetry of QCD in the background of magnetic field (131) regardless if the background magnetic field is a quantized (dynamical) field or a classical (static) field. 
The $\rho$-meson condensates (103) have the following general form

$$
\rho_{ \pm}(x)=\left\langle\bar{\psi}(x) \gamma_{ \pm} \tau_{ \pm} \psi(x)\right\rangle
$$

where $\psi=(u, d)^{T}$ is the quark spinor in Dirac, color and flavor space, $\gamma_{ \pm}=\left(\gamma_{1} \pm\right.$ $\left.i \gamma_{2}\right) / 2$ and $\tau_{ \pm}=\left(\tau_{1} \pm i \tau_{2}\right) / 2$ are combinations of the Dirac (spinor) and Pauli (flavor) matrices, respectively.

The condensates (136) are obviously invariant under the baryonic $U(1)_{B}$ transformations, $\psi \rightarrow e^{i \omega_{B}} \psi$, while the remaining global $U(1)_{I_{3}}$ group, $\psi \rightarrow e^{i \omega_{I_{3}} \tau_{3} / 2} \psi$, affects the vector condensates (136) as follows:

$$
U(1)_{I_{3}}: \quad \rho_{ \pm}(x) \rightarrow e^{\mp i \omega_{I_{3}}} \rho_{ \pm}(x) .
$$

It was noted in Ref. [85] that a possible spontaneous breaking of the $U(1)_{I_{3}}$ global symmetry (137) by the vector condensates (136) may contradict the Vafa-Witten theorem. This theorem implies that no massless Nambu-Goldstone boson associated with an internal symmetry breaking may occur in vector-like theories (for example, in QCD) with zero theta angle, ${ }^{86}$ so that vector-like global symmetries (like isospin or baryon number) cannot be spontaneously broken in QCD. However, as it was shown in Ref. [87], the massless boson does not emerge in the superconducting phase of QCD because the $U(1)_{I_{3}}$ global transformation (137) is, in fact, a part of the larger, electromagnetic symmetry group (135):

$$
U(1)_{\mathrm{em}}: \quad \rho_{ \pm}(x) \rightarrow e^{\mp i \omega_{\mathrm{em}}(x)} \rho_{ \pm}(x) .
$$

Equation (138) reflects the trivial fact that the vector quantities (136) are condensates of the electrically charged particles, so that they are sensitive to the electromagnetic $U(1)_{\mathrm{em}}$ transformation (135) as well. Thus, the condensates (136) break the internal local symmetry (135) and in this case the Nambu-Goldstone boson is known to be absent in agreement with the Vafa-Witten theorem: ${ }^{86}$ the would-be Nambu-Goldstone boson is absorbed into the Abelian gauge field $A_{\mu}^{\mathrm{em}}$ thus making the photon massive via a Higgs mechanism. A discussion of internal symmetries can also be found in Refs. [88, 89].

Let us now discuss external symmetries of the theory. In the presence of the background magnetic field $\boldsymbol{B}_{\text {ext }}$ the group of the space rotations $S O(3)_{\text {rot }}$ is explicitly broken down to its $O(2)_{\text {rot }}$ subgroup generated by rotations of space around the axis of the magnetic field. However, the presence of the intrinsic inhomogeneities of the $\rho$-meson condensate breaks spontaneously the residual rotational group $O(2)_{\text {rot }}$ further down to the group of discrete rotations of the vortex lattice. The translational group is also spontaneously broken by the inhomogeneous ground state. Thus, the spectrum of the QCD excitations should contain Nambu-Goldstone modes associated with spontaneous breaking of these external global symmetries of the QCD Lagrangian (131). The massless Nambu-Goldstone modes are elementary acoustic vibrations (phonons) of the vortex lattice in the ground state of the theory which are analogous to the acoustic modes in the mixed Abrikosov phase of the type-II superconductors. ${ }^{61}$ We discuss these modes in the next Section. 


\subsection{Beyond the mean field}

So far we have discussed the ground state using the mean-field approach to the effective bosonic model which describes electrodynamics of the point-like $\rho$ mesons. The quark-antiquark bound states are, in fact, extended objects and this fact may essentially influence their dynamics. Thus, the applicability of the effective bosonic model may be put to question. ${ }^{85}$ On the other hand, we know that the Ginzburg-Landau model describes very well, both qualitatively and in many cases quantitatively, all basic properties of the essentially nonlocal Cooper pairs using a local scalar field. Thus, despite of the nonlocal nature of the electrically charged quark-antiquark bound states, the local $\rho$-meson electrodynamics may capture important features of the $\rho$-meson condensation.

In addition, the $\rho$-meson condensation was also found in other approaches which are not based on a point-like assumption of the $\rho$ meson state at the level of fundamental fields. These approaches include the AdS/CFT treatment of the nonperturbative QCD in strong magnetic field ${ }^{40-43}$ as well as the methods based on the extended Nambu-Jona-Lasinio model. ${ }^{38,39}$ All of them are treating the ground state in certain form of a mean-field approximation, and all of them agree with the predictions based on the vector-meson dominance model (93). For the sake of completeness, we would like to discuss below other interesting issues of the $\rho$-meson condensation beyond the mean-field methods.

\subsubsection{Acoustic vibrations of the vortex lattice}

In the mean-field approximation the ground state of QCD in strong magnetic field is described by the hexagonal lattice of straight parallel vortex lines. This state corresponds to the minimum of the energy density. However, in a full theory the quantum fluctuations may lead to perturbations of the vortex lattices around their mean-field positions. Depending of the strength of these fluctuations the vortex lattice may melt and even evaporate. In the context of the solid state physics, the vortex lattice-liquid-gas phase diagram for a similar magnetic-field-induced (reentrant) superconductivity was discussed in Ref. [90].

The stability of the vortex lattice against perturbations depends also on the height of the potential energy barrier between different local conformations of the lattice. It turns out that this energy barrier is very small. For example, a difference in the mean energy between square and hexagonal lattices is less then $1 \%$ of the total condensation energy at $B=1.01 B_{c}^{\mathrm{QCD}}$. Thus, we expect that at moderate (not asymptotically high) values of the magnetic field the vortex lattice may be unstable against continuous conformations and eventual melting.

Acoustic vibrations of the vortex lattice are a simplest form of perturbations. The spectrum of the elementary acoustic excitations, phonons, was derived recently in Ref. [91]. Due to the geometrical structure of the ground state there are two types of phonons corresponding to longitudinal and transverse vibrations of the lattice. 
The dispersion relation for the low-energy phonons is:

$$
\begin{aligned}
\omega_{k}^{2} & =k_{z}^{2}+f(B)\left(\boldsymbol{k}_{\perp}^{2}\right)^{2}+\ldots, \\
f(B) & =\frac{C_{f}}{|e B|}\left(1-\frac{B_{c}^{\mathrm{QCD}}}{B}\right)+\ldots,
\end{aligned}
$$

where $k_{z}$ and $\boldsymbol{k}_{\perp}$ are the longitudinal and transverse momenta, respectively, $C_{f} \simeq$ 0.455 is a constant, and the higher-order corrections in $\boldsymbol{k}_{\perp}^{2}$ and in $B-B_{c}^{\mathrm{QCD}}$ are shown by the ellipsis. This expansion is valid in the vicinity of the transition point $B \geqslant B_{c}^{\mathrm{QCD}}$.

Apart from the prefactor, the dispersion relation (139) for the low-energy phonons in the $\rho$-vortex ground state has the same qualitative form as the dispersion relation for the acoustic phonons in Abrikosov vortex lattices in conventional superconductors.

At $\boldsymbol{k}_{\perp}=0$ the dispersion relation (139) for the acoustic vibrations of the vortex lattice along the direction of the magnetic field is a linear function of momentum. A phonon propagating in the transverse plane possesses a quadratic (supersoft) dispersion relation in the limit of small momenta. Thus, the longitudinal phonons propagate with the speed of light while the velocity of the transverse acoustic phonons, $v_{\perp}\left(\boldsymbol{k}_{\perp}, k_{z}=0\right)=2 \sqrt{f(B)}\left|\boldsymbol{k}_{\perp}\right|$, depends on their energy. For example, at $B=1.01 B_{c}^{\mathrm{QCD}}$ a transverse acoustic phonon carrying energy $\omega_{\boldsymbol{k}, 0}=1 \mathrm{MeV}$ should propagate with the velocity equal to $2 \%$ of the speed of light.

The presence of the supersoft (quadratic) transversal phonon modes is known to be crucial for the stability of the vortex lattice since these modes make an infrared divergent contribution to the free energy of the system. ${ }^{61}$ As a result, the vortex lattice may become unstable and melt into a vortex liquid. The vortex liquid state was indeed observed in numerical simulations of quenched QCD. ${ }^{92}$ We discuss it in the next section.

\subsubsection{Melting of the vortex lattice: results of numerical simulations}

The vortices were visualized in numerical Monte-Carlo simulations of quenched QCD with two colors ${ }^{44}$ in a background of strong magnetic field. Although the $\rho$ meson condensate cannot be computed directly in this simplest approach, it can be accessed via the following $\rho$-meson correlator:

$$
\phi(x)=\left\langle\rho^{\dagger}(0) \rho(x)\right\rangle
$$

where the $\rho$ meson field is defined by Eq. (103). The correlation function $\phi(x)$ is computed in the fixed background of both the non-Abelian gauge field and the Abelian magnetic field. We refer an interested reader to Ref. [44] for further technical details.

Under the electromagnetic gauge group $U(1)_{\text {e.m. }}$ the field (141) transforms as a charged scalar field ${ }^{\mathrm{b}}: \phi(x) \rightarrow e^{i e \omega(x)} \phi(x)$. However, the effective field $\phi(x)$ is

\footnotetext{
b The gauge transformation at the origin, $\varphi(x) \rightarrow e^{i e \omega(0)} \varphi(x)$, acts as a global phase which is not
} 
determined by a two-point correlation function which falls off exponentially as the distance $x$ increases. This property is not a desired behavior for a genuine local scalar field so that, obviously, the quantity (141) cannot be associated with the $\rho$-meson field itself.

Fortunately, we may get an insight from Ref. [93] where a qualitatively similar problem was encountered in a different physical setup. In Ref. [93] the chromoelectric flux tube was studied using a rectangular Wilson loop $\mathcal{W}$ as a source and the local energy density operator $\mathcal{O}$ as a probe. Although the expectation value of the Wilson loop falls off exponentially as the area of the Wilson loop grows, the energy density in the presence of the Wilson loop, given by the normalized energy ratio $\langle\mathcal{O}\rangle_{\mathcal{W}}=\langle\mathcal{O} \mathcal{W}\rangle /\langle\mathcal{W}\rangle$, has, generally, a non-vanishing profile as the area of the Wilson loop grows. The study of the normalized energy ratio was very useful in a visualization of the confining QCD flux tube which could not be accessed by other nonperturbative lattice methods.

By analogy with Ref. [93], one can consider the normalized Abelian scalar energy of the $\rho$-meson field in transversal $(x, y)$ plane: ${ }^{44}$

$$
E(x)=\frac{\left|D_{\mu} \phi(x)\right|^{2}}{|\phi(x)|^{2}}, \quad D_{\mu}=\partial_{\mu}-i e A_{\mu}
$$

where we used the continuum notations for the sake of simplicity. Since the vortices carry a certain energy, their positions should be seen as one-dimensional objects in three dimensional coordinate space characterized by an elevated energy density.
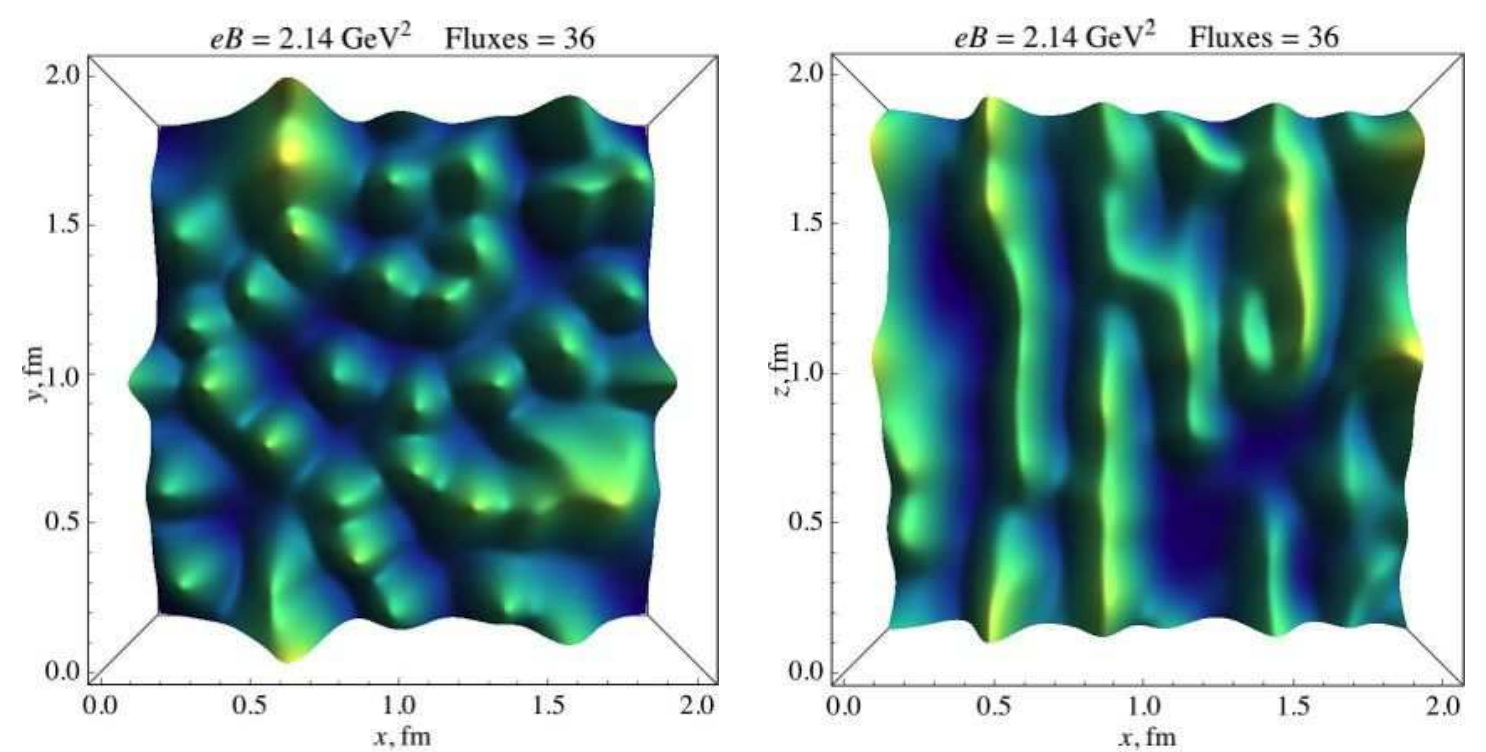

Fig. 17. A on-top view of the typical behavior of the Abelian energy density (142) in (left) the $(x, y)$ plane and (right) the $(x, z)$ plane for magnetic field $e B=2.14 \mathrm{GeV}^{2}$. The energy density was interpolated for a better visualization. In the left plot the magnetic field is perpendicular to the page while in the right plot the magnetic field is directed vertically (from Ref. [91]).

essential for our interpretation of the effective field $\phi(x)$. 
The examples of a typical behavior of the energy density (142) which arise in the numerical simulations of quenched lattice QCD are shown in Fig. 17. The positions of the vortices are clearly seen in the transverse crosssection of the field configuration, Fig. 17(left). However, instead of a regular lattice of vortices - predicted by the mean-field theory - we observe an unordered ensemble of the vortex cores. This picture reminds us a disordered liquid rather then an ordered lattice of vortices.

In the longitudinal crosssection, Fig. 17(right), the vortices are seen as elongated objects mostly parallel to magnetic field. However, even in this case one observes that the vortices are not straight parallel lines as they may disappear by shifting to another slice. Thus, the lattice simulations reveal that the vortices do not form a regular vortex lattice, at least in quenched numerical simulations of QCD. Moreover, two-point correlation functions of the vortex densities suggest that in strong magnetic field the vortex ensembles are better described by a liquid rather than by a solid lattice. ${ }^{44}$ This numerical result is in a qualitative agreement with the existence of the destructive ultrasoft phonon modes discussed above.

\subsubsection{Nature of the transition to the superconducting ground state}

The evidence of the existence of the $\rho$ vortices in lattice simulations points out that a new superconducting ground state is formed at strong magnetic field. ${ }^{44}$ All known mean-field methods, which utilize the effective electrodynamics of $\rho$ mesons, ${ }^{35,36}$ holographic methods ${ }^{40-43}$ and Nambu-Jona-Lasinio approaches ${ }^{38,39}$ suggest that the phase transition should be of second order. On the other hand, lattice simulations with dynamical fermions indicate that at low temperature a representative set of observables do not have any noticeable phase singularities at a wide range of strengths of the magnetic field. ${ }^{27}$ Moreover, it was found in quenched lattice simulations that the $\rho$-meson mass is a nonmonotonic function of magnetic field: with increase of the magnetic field strength the mass decreases up to a nonzero value and then start to increase again. All these facts indicate that the phase transition may not be of the second order, and a weak crossover may take place instead.

In general, one may expect that the inclusion of the quantum fluctuations may enhance or weaken the mean-field transition making it either a first order transition or a crossover, respectively. Notice that in the cases of the first-order transition and crossover the $\rho$-meson mass should not be vanishing at the transition point. An illustration of a generic behavior of the lowest mass for all these transitions is shown in Fig. 18.

For example, both first, second and crossover transitions are realized in the electroweak model at a finite temperature. The strength of the transition depends on the value of the zero-temperature Higgs mass. In this model the behavior of the lowest (scalar) mass on temperature $T$ follows Fig. 18 (with $X \equiv T$ ). ${ }^{94}$ The behavior of the $\rho$-meson mass in QCD vs. magnetic field is qualitatively described by the dashed line in Fig. 18 (in this case $X \equiv e B_{\text {ext }}$ ).

A direct calculation of the $\rho$-meson condensate in lattice calculation is quite 


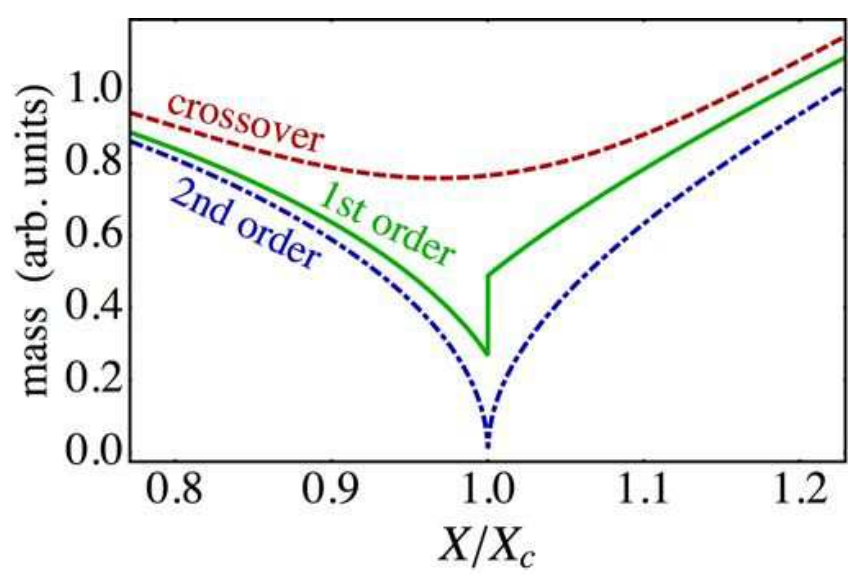

Fig. 18. Qualitative behavior of a mass of a lowest excitation associated with an order parameter in a generic system as a function of a thermodynamic parameter $X$ (magnetic field $B$, temperature $T$ etc) for a first and second order transitions and for a crossover.

difficult due the strong inhomogeneities of the condensate. As one can see from Fig. 13(right) the phase (and the sign) of the condensate in the ground state is a lively function of the transverse coordinates. This is the reason why the bulk average of the $\rho$ condensate (24), (25), (37) and (114) over the whole transverse $x_{\perp} \equiv\left(x_{1}, x_{2}\right)$ plane should always be zero,

$$
\langle\rho\rangle \equiv\left\langle\frac{1}{\mathrm{Vol}_{\perp}} \int d^{2} x_{\perp} \rho(x)\right\rangle \equiv\left\langle\frac{1}{\mathrm{Vol}} \int d^{4} x \rho(x)\right\rangle \equiv 0 .
$$

Due to the translational symmetry of QCD, Eq. (143) implies that the expectation value of the local operator $\rho(x)$ should also be vanishing in a finite physical volume. Indeed, all coordinate-shifted copies of any field configuration enter the partition function with the same weight so that the vacuum expectation value (v.e.v.) of the local field operator $\langle\rho(x)\rangle$ is equal to the v.e.v. of its average over the whole space. The latter is zero (143) in agreement with the Elitzur's theorem. ${ }^{95}$ Thus, on a practical side, $\langle\rho(x)\rangle$ is not a good local order of the inhomogeneous $\rho$-meson condensation. Notice that the homogeneous condensate of $\rho$ mesons in QCD is ruled out both by Ref. [85] and Ref. [38]. The very same property (143) is shared by the celebrated Abrikosov vortex lattices in type-II superconductors: ${ }^{62}$ despite the vortex-lattice ground state is a superconducting state with a locally large order parameter (24), the bulk average of the corresponding order parameter is nevertheless vanishing due to the unavoidable presence of the Abrikosov vortices.

In fact, the mean value of the inhomogeneous condensate vanishes quickly with the increase of the transverse area $L_{\perp}^{2}$. In the large-volume limit $L_{\perp} \gg L_{B}$ the average of the inhomogeneous condensate has the following asymptotic behavior: $\left|\langle\rho\rangle_{L_{\perp}}\right|=\frac{L_{0}}{L_{\perp}^{2}}+O\left(L_{\perp}^{-4}\right)$, where $L_{0}=\alpha L_{B} \rho_{\infty}$ and $\alpha \approx 3.27$ is a numerical constant associated with the geometry of the $\rho$-vortex lattice. ${ }^{89}$ Thus the expectation value of the $\rho$-meson field is not able to reveal the presence in the thermodynamic limit.

A two-point correlation function of $\rho$-meson operators shows a presence of the condensate given by a plateau in the correlation functions at moderate separations 
between the points. ${ }^{44}$ There is evidence, that at larger separations the plateau disappears. ${ }^{85}$ This property is consistent with the vibrations of the $\rho$ vortices in a liquid vortex phase. ${ }^{89}$

Thus, the results of numerical simulations are consistent with the existence of a weak crossover transition. The formation of the $\rho$ vortices was observed in lattice QCD in a strong magnetic field background. However, the vortex ground state lacks the lattice order predicted by the mean-field methods. Instead, the numerical simulations of QCD point out to existence of a vortex liquid phase at strong magnetic field.

\section{Conclusions}

We have discussed superconducting phases which may emerge in the vacuum of the Standard Model in strong magnetic field background at zero temperature. The superconductivity - which is understood in the usual electromagnetic sense - is mediated by condensation of electrically charged vector degrees of freedom.

There are two suggested superconducting phases in the Standard Model. In strong magnetic fields of the QCD scale, $B \sim 10^{16} \mathrm{~T}$, the superconductivity is associated with condensation of quark-antiquark pairs with the $\rho$ meson quantum numbers. In stronger magnetic fields of the electroweak scale, $B \sim 10^{20} \mathrm{~T}$, the superconducting phase emerges as a result of condensation of the $W$ bosons.

We have described the following basic properties of the superconducting states:

(1) The superconducting effect occurs because of the nonminimal coupling of the charged vector particles $\left(\rho^{ \pm}\right.$mesons in QCD and $W$ bosons in electroweak model) to the electromagnetic field. The strong magnetic field enhances the electromagnetic superconductivity of the vacuum instead of destroying it.

(2) The superconducting state is anisotropic: the electric resistance is zero only along the axis of the magnetic field.

(3) The superconducting state is always inhomogeneous: the condensate shares similarity with the Abrikosov vortex lattice in the mixed state of a type-II superconductor. A spatially homogeneous superconducting state does not exist.

(4) The onset of the superconductivity of the charged particles ( $\rho^{ \pm}$mesons in QCD and $W$ bosons in electroweak model) leads to emergence of an inhomogeneous superfluidity of the neutral degrees of freedom $\left(\rho^{0}\right.$ mesons in QCD and $Z$ bosons in electroweak model). The superfluidity is induced by the inhomogeneities of the corresponding superconducting condensate.

(5) The Meissner effect cannot be realized in the superconducting state due to anisotropic nature of the magnetic-field-induced superconductivity.

(6) In a mean-field approximation the inhomogeneous superconducting state is realized as a vortex lattice. The vortices are parallel to the magnetic-field axis and they form a regular lattice structure in a transversal plane.

(7) The mean-field vortex ground state has a "kaleidoscopic" lattice structure made of the equilateral triangular lattice of the superconductor vortices which is su- 
perimposed on the hexagonal lattice of the superfluid vortices.

(8) Locally, the vortex cores expel both superconducting and superfluid condensates. A unit vortex cell carries one unit of the quantized magnetic flux of the magnetic field while the flux corresponding to the neutral particles is vanishing.

(9) The inhomogeneous superconducting condensate breaks spontaneously rotational and translational symmetries of the corresponding theories leading to emergence of acoustic phonons. The phonons play a role of the NambuGoldstone bosons associated with vibrations of the vortex lattice.

(10) Due to the presence of the supersoft acoustic modes the regular vortex lattice may melt into a less ordered vortex liquid. Consequently, the transition to the superconducting phase may be very smooth similarly to a water gas-liquid transition above a critical endpoint.

(11) Preliminary first-principle results coming from numerical simulations of lattice QCD are compatible with existence of a very smooth crossover transition from the insulating hadronic phase at low magnetic field to the superconducting phase at strong magnetic field. Numerical investigation of the superconducting phase in the electroweak sector of the Standard Model are not available yet.

There are also many interesting open problems such as observation of vortex lattice/liquid state and numerical calculation of conductivity in simulations both in lattice QCD with dynamical fermions and in the bosonic sector of the electroweak model, extension of the analysis of the superconducting ground state beyond the existing mean-field approaches, analytical exploration of QCD in asymptotic limit of high magnetic fields, study of thermal effects etc. The magnetic-field-induced superconductivity of the vacuum is an intriguing, counterintuitive and yet-unexplored field-theoretical phenomenon which deserves further study.

\section{Acknowledgments}

The work was partially supported by grant No. ANR-10-JCJC-0408 HYPERMAG of Agence Nationale de la Recherche (France). The author thanks Jor Van Doorsselaere for careful reading of the manuscript and helpful comments.

\section{References}

1. S. L. Adler, Annals Phys. 67, 599 (1971).

2. R. Battesti and C. Rizzo, Rept. Prog. Phys. 76, 016401 (2013).

3. V. Skokov, A. Y. Illarionov, V. Toneev, Int. J. Mod. Phys. A24, 5925 (2009).

4. A. Bzdak and V. Skokov, Phys. Lett. B 710, 171 (2012).

5. W.-T. Deng and X. -G. Huang, Phys. Rev. C 85, 044907 (2012);

6. D. Kharzeev, Phys. Lett. B 633, 260 (2006).

7. D. Grasso, H. R. Rubinstein, Phys. Rept. 348, 163 (2001).

8. K. Fukushima, D. E. Kharzeev, H. J. Warringa, Phys. Rev. D78, 074033 (2008);

9. D. E. Kharzeev, L. D. McLerran and H. J. Warringa, Nucl. Phys. A 803, 227 (2008).

10. A. Vilenkin, Phys. Rev. D 22, 3080 (1980);

11. M. A. Metlitski and A. R. Zhitnitsky, Phys. Rev. D 72, 045011 (2005). 
12. S. A. Voloshin, Phys. Rev. C 70, 057901 (2004).

13. B. I. Abelev et al. [STAR Collaboration], Phys. Rev. Lett. 103, 251601 (2009).

14. B. I. Abelev et al. [STAR Collaboration], Phys. Rev. C 81, 054908 (2010)

15. B. Abelev et al. [ALICE Collaboration], Phys. Rev. Lett. 110, 012301 (2013).

16. H. Suganuma and T. Tatsumi, Annals Phys. 208, 470 (1991).

17. K. G. Klimenko, Z. Phys. C 54, 323 (1992);

18. V. P. Gusynin, V. A. Miransky, I. A. Shovkovy, Phys. Rev. Lett. 73, 3499 (1994).

19. V. P. Gusynin, V. A. Miransky, I. A. Shovkovy, Phys. Lett. B 349, 477 (1995).

20. V. P. Gusynin, V. A. Miransky, I. A. Shovkovy, Nucl. Phys. B462, 249 (1996).

21. R. Gatto and M. Ruggieri, Phys. Rev. D 83, 034016 (2011).

22. R. Gatto and M. Ruggieri, Phys. Rev. D 82, 054027 (2010).

23. E. S. Fraga and A. J. Mizher, Phys. Rev. D 78, 025016 (2008).

24. A. J. Mizher, M. N. Chernodub and E. S. Fraga, Phys. Rev. D 82, 105016 (2010).

25. M. D'Elia, S. Mukherjee and F. Sanfilippo, Phys. Rev. D 82, 051501 (2010).

26. E. S. Fraga and L. F. Palhares, Phys. Rev. D 86, 016008 (2012).

27. G. S. Bali, F. Bruckmann, G. Endrodi, Z. Fodor, S. D. Katz, S. Krieg, A. Schafer, K. K. Szabo, JHEP 1202, 044 (2012).

28. F. Bruckmann, G. Endrodi and T. G. Kovacs, JHEP 1304, 112 (2013).

29. E. J. Ferrer, V. de la Incera and C. Manuel, Phys. Rev. Lett. 95, 152002 (2005).

30. S. Fayazbakhsh and N. Sadooghi, Phys. Rev. D 82, 045010 (2010).

31. A. Rabhi, P. K. Panda and C. Providencia, Phys. Rev. C 84, 035803 (2011);

32. A. A. Isayev and J. Yang, Phys. Rev. C 84, 065802 (2011).

33. F. Preis, A. Rebhan and A. Schmitt, J. Phys. G 39, 054006 (2012).

34. V. Dexheimer, R. Negreiros and S. Schramm, Eur. Phys. J. A 48, 189 (2012).

35. M. N. Chernodub, Phys. Rev. D82, 085011 (2010).

36. M. N. Chernodub, J. Van Doorsselaere and H. Verschelde, Phys. Rev. D 85, 045002 (2012).

37. I. I. Smolyaninov, Phys. Rev. Lett. 107, 253903 (2011).

38. M. N. Chernodub, Phys. Rev. Lett. 106, 142003 (2011).

39. M. Frasca, JHEP 1311, 099 (2013).

40. M. Ammon, J. Erdmenger, P. Kerner and M. Strydom, Phys. Lett. B 706, 94 (2011).

41. Y.-Y. Bu, J. Erdmenger, J. P. Shock and M. Strydom, JHEP 1303, 165 (2013).

42. N. Callebaut, D. Dudal and H. Verschelde, JHEP 1303, 033 (2013).

43. N. Callebaut and D. Dudal, JHEP 1401, 055 (2014).

44. V. V. Braguta, P. V. Buividovich, M. N. Chernodub, A. Y. .Kotov and M. I. Polikarpov, Phys. Lett. B 718, 667 (2012).

45. E. V. Luschevskaya and O. V. Larina, JETP Lett. 98, 652 (2014).

46. J. Ambjorn and P. Olesen, Nucl. Phys. B 315, 606 (1989).

47. Phys. Lett. B 218, 67 (1989).

48. Int. J. Mod. Phys. A 5, 4525 (1990).

49. P. Olesen, arXiv:1311.4519 [hep-th].

50. R. -G. Cai, S. He, L. Li and L. -F. Li, JHEP 1312, 036 (2013).

51. R. -G. Cai, L. Li, L. -F. Li and Y. Wu, JHEP 1401, 045 (2014).

52. R. -G. Cai, L. Li and L. -F. Li, JHEP 1401, 032 (2014).

53. S. W. MacDowell and O. Tornkvist, Phys. Rev. D 45, 3833 (1992).

54. M. N. Chernodub, J. Van Doorsselaere and H. Verschelde, Phys. Rev. D 88, 065006 (2013).

55. J. Van Doorsselaere, Phys. Rev. D 88, no. 2, 025013 (2013).

56. N. K. Nielsen and P. Olesen, Nucl. Phys. B 144, 376 (1978).

57. J. Ambjorn and P. Olesen, Nucl. Phys. B 170, 60 (1980). 
58. J. Ambjorn and P. Olesen, Nucl. Phys. B 170, 265 (1980).

59. P. Olesen, Phys. Lett. B 268, 389 (1991).

60. M. Tinkham, Introduction to Superconductivity (Dover Publications, New York, 2004).

61. B. Rosenstein and D. Li, Rev. Mod. Phys. 82, 109 (2010).

62. A. A. Abrikosov, Sov. Phys. JETP 5, 1174 (1957).

63. E. B. Bogomolny, Sov. J. Nucl. Phys. 24, 449 (1976) [Yad. Fiz. 24, 861 (1976)].

64. M. Rasolt, Phys. Rev. Lett. 58, 1482 (1987).

65. Z. Tešanović, M. Rasolt, L. Xing, Phys. Rev. Lett., 632425 (1989).

66. M. Rasolt, Z. Tešanović, Rev. Mod. Phys. 64, 709 (1992).

67. F. Lévy, I. Sheikin, B. Grenier, A. D. Huxley, Science 309, 1343 (2005).

68. D. Aoki, T. D. Matsuda, V. Taufour, E. Hassinger, G. Knebel, J. Flouquet, J. Phys. Soc. Jpn. 78 (2009) 113709.

69. A. Salam and J. A. Strathdee, Nucl. Phys. B 90, 203 (1975).

70. A. D. Linde, Phys. Lett. B 62, 435 (1976).

71. G. Aad et al. (ATLAS Collaboration), Phys. Lett. B 716, 1 (2012).

72. S. Chatrchyan et al. (CMS Collaboration), Phys. Lett. B 716, 30 (2012).

73. See, e.g., the review by A. Achucarro and T. Vachaspati, Phys. Rept. 327, 347 (2000).

74. M. S. Volkov, Phys. Lett. B 644, 203 (2007).

75. J. Garaud and M. S. Volkov, Nucl. Phys. B 826, 174 (2010).

76. N. K. Nielsen and P. Olesen, Phys. Lett. B 79, 304 (1978).

77. H. B. Nielsen and P. Olesen, Nucl. Phys. B 160, 380 (1979).

78. G. K. Savvidy, Phys. Lett. B 71, 133 (1977).

79. Y. Nambu and G. Jona-Lasinio, Phys. Rev. 122, 345 (1961).

80. S. Schramm, B. Muller and A. J. Schramm, Mod. Phys. Lett. A 7, 973 (1992).

81. D. Djukanovic, M. R. Schindler, J. Gegelia, S. Scherer, Phys. Rev. Lett. 95, 012001 (2005).

82. J. J. Sakurai, Annals Phys. 11, 1 (1960).

83. J. Beringer et al. (Particle Data Group), Phys. Rev. D86, 010001 (2012).

84. N. M. Kroll, T. D. Lee and B. Zumino, Phys. Rev. 157, 1376 (1967).

85. Y. Hidaka and A. Yamamoto, Phys. Rev. D 87, 094502 (2013).

86. C. Vafa and E. Witten, Nucl. Phys. B 234, 173 (1984).

87. M. N. Chernodub, Phys. Rev. D 86, 107703 (2012).

88. C. Li and Q. Wang, Phys. Lett. B 721, 141 (2013).

89. M. N. Chernodub, Phys. Rev. D 89, 018501 (2014).

90. Z. Tešanović, Phys. Rev. B 596449 (1999).

91. M. N. Chernodub, J. Van Doorsselaere and H. Verschelde, arXiv:1401.0264 [hep-ph].

92. V. V. Braguta, P. V. Buividovich, M. Chernodub, M. I. Polikarpov and A. Y. Kotov, PoS ConfinementX, 083 (2012).

93. G. S. Bali, K. Schilling and C. Schlichter, Phys. Rev. D 51, 5165 (1995).

94. K. Kajantie, M. Laine, K. Rummukainen and M. E. Shaposhnikov, Phys. Rev. Lett. 77, 2887 (1996); an extended version of the paper is available as hep-ph/9605288.

95. S. Elitzur, Phys. Rev. D 12, 3978 (1975). 